\title{
Frame covariance in quantum gravity
}

\author{
Kieran Finn $\odot,{ }^{1}$ Sotirios Karamitsos, ${ }^{1,2}$ and Apostolos Pilaftsis $\odot^{1}$ \\ ${ }^{1}$ School of Physics and Astronomy, University of Manchester, Manchester M13 9PL, United Kingdom \\ ${ }^{2}$ Consortium for Fundamental Physics, Physics Department, Lancaster University, \\ Lancaster LA1 4YB, United Kingdom
}

(Received 11 March 2020; revised 29 June 2020; accepted 4 August 2020; published 20 August 2020)

\begin{abstract}
We develop a quantum effective action for scalar-tensor theories of gravity which is both spacetimediffeomorphism invariant and field reparametrization (frame) invariant beyond the classical approximation. We achieve this by extending the Vilkovisky-DeWitt formalism, treating both the scalar fields and the components of the gravitational tensor field as coordinates describing a manifold. By using tensors covariant under diffeomorphisms of this manifold, we show that scalar-tensor theories can be written in a form that is manifestly frame invariant at both classical and quantum levels. In the same context, we show that in order to maintain manifest frame invariance, we must modify the Feynman rules of theories with a nontrivial field space. We show that one such theory is general relativity by demonstrating explicitly that it has a nonzero field-space Riemann tensor. Thus, when constructing theories of quantum gravity, we must deal not only with curved spacetime, but also with a curved field space. Finally, we address the cosmological frame problem by tracing its origin to the existence of a new model function that appears in the path-integral measure. Once this function is fixed, we find that frame transformations have no effect on the quantization of the theory. The uniqueness of our improved quantum effective action is discussed.
\end{abstract}

DOI: 10.1103/PhysRevD.102.045014

\section{INTRODUCTION}

The laws of nature should not depend on the way we choose to describe them. While there may be many different ways of parametrizing the underlying degrees of freedom in a theory, its physical predictions should not depend on which parametrization one uses. This seemingly obvious fact has historically had far-reaching consequences. For example, imposing that the laws of physics not care about the way we label space and time leads inevitably to Einstein's celebrated theory of relativity [1]. This idea is known as reparametrization invariance and throughout this paper we use it as a guiding light with the goal of developing a formalism in which reparametrization invariance is made manifest.

When writing down a quantum field theory (QFT), we must define a set of quantum fields in which to express it. We are always free to reexpress the same theory in terms of a different set of fields. This is known as a change of frame. There has been much debate in the literature [2-19] as to whether such a change of frame represents an observable change to the theory or merely a change of description.

Published by the American Physical Society under the terms of the Creative Commons Attribution 4.0 International license. Further distribution of this work must maintain attribution to the author(s) and the published article's title, journal citation, and DOI. Funded by SCOAP ${ }^{3}$.
Since changes of frame correspond to field reparametrizations, we expect that they should not affect any physical observables. However, in the ordinary formulation of QFTs, off-shell calculations of quantum corrections can yield different results depending on the set of fields used to perform them, as shown in the Appendix A. Furthermore, it has been shown that when gravity is included, one can get different predictions even for on-shell observables depending on whether the quantum effects are applied before or after changing frame. This has become known as the cosmological frame problem. For a historical overview of the issue, see [20].

In light of the above issues, our aim is to develop a formalism in which reparametrization invariance is made manifest both on and off shell and hence does not suffer from the cosmological frame problem. It is important to note that whether or not a formalism is reparametrization invariant is a consequence of its representation, not its content. Any physical observable of the theory must be invariant under reparametrizations, but this fact can often be obscured by the way the theory is written down.

We emphasize that because the content of a theory has no bearing on reparametrization invariance, this formalism places no restrictions on which theories are allowed. Therefore, reparametrization invariance cannot be considered a symmetry in the traditional sense and there will, in general, be no Noether current and no gauge degrees of freedom associated with it. 
A better point of comparison is the use of spacetime tensors to highlight the diffeomorphism invariance of a QFT. Although the physical predictions of any theory will necessarily be independent of the spacetime coordinates used to perform the calculation, the use of covariant objects makes this fact manifest.

In this paper, we shall focus on scalar-tensor theories of quantum gravity [21-27] with a field content that consists of a spin-2 graviton field $g_{\mu \nu}$ and a set of scalar fields $\phi^{A}$ (collectively denoted as $\phi$ ) and with an action of the form

$$
\begin{aligned}
S & \equiv \int d^{4} x \sqrt{-g} \mathcal{L} \\
\mathcal{L} & \equiv-\frac{f(\boldsymbol{\phi})}{2} R+\frac{1}{2} g^{\mu \nu} k_{A B}(\boldsymbol{\phi}) \partial_{\mu} \phi^{A} \partial_{\nu} \phi^{B}-V(\boldsymbol{\phi}),
\end{aligned}
$$

where $g \equiv \operatorname{det}\left(g_{\mu \nu}\right)$. Here $f(\boldsymbol{\phi}), k_{A B}(\boldsymbol{\phi})$ and $V(\boldsymbol{\phi})$ are the effective Planck mass, the scalar field-space metric and the potential, respectively. We shall refer to these three functions as model functions and together they fully define our theory at the classical level. In the context of such theories, there are two types of transformations that amount to nothing more than a change of description-spacetime diffeomorphisms and field reparametrizations. We wish to write our theory in way that is manifestly invariant under both of these.

Spacetime diffeomorphisms consist of changing the coordinates of spacetime,

$$
x^{\mu} \rightarrow \tilde{x}^{\mu}=\tilde{x}^{\mu}\left(x^{\mu}\right) .
$$

This is just a relabeling of the points on the spacetime manifold and thus should not affect any physical observables. Diffeomorphism invariance is the backbone of general relativity and, as such, has been much studied in the literature. We will therefore not focus on it here.

Field reparametrizations involve changing the definition of the fields of the theory by making the transformation

$$
\begin{aligned}
& g_{\mu \nu} \rightarrow \tilde{g}_{\mu \nu}=\tilde{g}_{\mu \nu}\left(g_{\rho \sigma}, \boldsymbol{\phi}\right), \\
& \phi^{A} \rightarrow \tilde{\phi}^{A}=\tilde{\phi}^{A}\left(g_{\rho \sigma}, \boldsymbol{\phi}\right) .
\end{aligned}
$$

Again, this is just a relabeling of the degrees of freedom in the theory and should not have a physical effect.

Spacetime-diffeomorphism invariance restricts the class of field redefinitions that we have to consider. When performing the transformation (1.3), we must maintain the spacetime covariant structure of the fields and should not introduce any new spacetime tensors. If we also insist that our field redefinitions do not mix derivative and nonderivative terms, then this restricts the admissible set of transformations to those of the form

$$
g_{\mu \nu} \rightarrow \tilde{g}_{\mu \nu}=\Omega^{2}(\boldsymbol{\phi}) g_{\mu \nu},
$$

$$
\phi^{A} \rightarrow \tilde{\phi}^{A}=\tilde{\phi}^{A}(\boldsymbol{\phi}) .
$$

We will refer to these transformations as a conformal transformation and a scalar field reparametrization, respectively. Together, they constitute a frame transformation. Under such a frame transformation, the model functions in (1.1) transform as [28]

$$
\begin{aligned}
f \rightarrow \tilde{f}= & \Omega^{-2} f, \\
V \rightarrow \tilde{V}= & \Omega^{-4} V \\
k_{A B} \rightarrow \tilde{k}_{A B}= & K^{C}{ }_{A} K^{D}{ }_{B}\left[k_{C D}-6 f(\ln \Omega)_{, C}(\ln \Omega)_{, D}\right. \\
& \left.+3 f_{, C}(\ln \Omega)_{, D}+3(\ln \Omega)_{, C} f_{, D}\right],
\end{aligned}
$$

where a comma ${ }_{A} \equiv \partial / \partial \phi^{A}$ denotes differentiation with respect to the field $\phi^{A}$ and $K_{B}^{A} \equiv \partial \phi^{A} / \partial \tilde{\phi}^{B}$ is the Jacobian of the scalar field reparametrization.

In this paper, we will show explicitly how we can write down a theory in a manifestly reparametrization-invariant way by using the well-known technique of field-space covariance [29-33], whose relevance to resolving the cosmological frame problem was first pointed out in [34]. We treat both the scalar fields and the components of the graviton field as coordinates describing a manifold. Frame transformations of the form (1.3) are then simply diffeomorphisms of this manifold. Provided we write down our theory in terms of objects that are both spacetime and field-space tensors, and then fully contract any indices, the theory will be manifestly reparametrization invariant.

With the field-space covariant technique, the theory of general relativity (which is just a scalar-tensor theory without the scalars) can also be expressed in terms of a field-space manifold. This manifold is separate from the spacetime manifold and comes with its own Riemann tensor, Ricci tensor, and Ricci scalar. As we will see in Sec. VI, all these curvature invariants are nonzero. Thus, when studying quantum theories of gravity, we must necessarily deal not only with curved spacetime, but with a curved field space as well. We believe that this observation will be important to consider when constructing a UV complete theory of quantum gravity and may be part of the reason why such a construction has proven so difficult.

We shall express our reparametrization-invariant theory using the quantum effective action [35-38]. All predictions of the theory can be obtained from this effective action and thus defining it is sufficient to fully define the quantum theory. However, as we shall see in Sec. III, the ordinary construction of the effective action depends on our choice of parametrization.

If we can treat gravity as a classical background, the Vilkovisky-DeWitt (VDW) formalism $[39,40]$, reviewed in Sec. IV, is enough to solve this problem. However, if we wish to treat gravity as a field and place it on the same footing as the other fields in our theory, we encounter 
ambiguities, which inevitably lead to the cosmological frame problem.

As we shall show, these ambiguities arise from a framedependent choice that must be made in the standard approach to scalar-tensor theories of gravity. The graviton field $g_{\mu \nu}$ is normally identified as the metric of spacetime. However, $g_{\mu \nu}$ transforms under a field reparametrization (1.3) whereas the metric of spacetime does not. This identification is therefore only valid in a particular frame [17], and thus the frame invariance of the VDW formalism is ruined.

In this paper, we overcome the cosmological frame problem by defining the metric of spacetime in a frameinvariant manner. We achieve this through the introduction of a new model function, $\ell=\ell(\boldsymbol{\phi})$, so that the metric of spacetime is given by $\bar{g}_{\mu \nu}=g_{\mu \nu} / \ell^{2}$. We are therefore able to construct, for the first time, a manifestly frame and spacetime-diffeomorphism-invariant quantum effective action for scalar-tensor theories of gravity.

In practice, the quantum effects of a theory are usually calculated using Feynman diagrams. However, as we shall see in Sec. V, the usual way in which these diagrams are calculated crucially depends on the frame in which they are evaluated. Feynman rules, when calculated in the usual way, are not covariant field-space tensors and thus different parametrizations of the fields will yield different sets of rules. We will show how the Feynman rules must be modified in the presence of a nontrivial field space.

We adopt the following conventions throughout this paper. Lowercase Greek letters ( $\mu, \nu$, etc.) will be used for spacetime indices, and repeated indices will imply summation in accordance with the Einstein summation convention. Uppercase Latin letters ( $A, B$, etc.) will be used for field-space indices with repeated indices again implying summation. Lowercase Latin letters $(a, b$, etc.) will be used for configuration-space indices and will thus simultaneously represent both a discrete field-space index and a point in spacetime. For such indices, we shall use the EinsteinDeWitt notation [41] in which repeated configuration-space indices imply summation over the discrete index and integration over spacetime, e.g.,

$$
J_{a} \phi^{a} \equiv \sum_{A} \int d^{D} x_{A} \sqrt{-\bar{g}} J_{A}\left(x_{A}\right) \phi^{A}\left(x_{A}\right)
$$

where $D$ is the number of spacetime dimensions and $\bar{g}_{\mu \nu}$, with determinant $\bar{g}$, is the metric of spacetime.

This paper is laid out as follows. We begin in Sec. II by reviewing the construction of the field and configuration spaces for scalar field theories. We then review the effective action formalism in Sec. III, explicitly demonstrating that it is dependent on the parametrization of the fields. We show in Sec. IV how Vilkovisky and DeWitt's reformulated effective action resolves these issues when gravity can be treated as a background. In Sec. V, we show the effect of reparametrizations on ordinary quantum calculations using Feynman diagrams and develop a method for calculating Feynman rules in a reparametrization-invariant manner.

We show how the same geometric approach of Vilkovisky and DeWitt can be applied to gravity in Sec. VI, explicitly constructing the field space for general relativity and showing that this field space is positively curved. We add scalar fields to the theory in Sec. VII, showing that when we do, there is an ambiguity in the definition of the spacetime metric, which is responsible for the cosmological frame problem. In Sec. VIII, we construct a field space for the scalar and tensor fields, which we call the grand field space, and use it to write down scalar-tensor theories in a way that is manifestly invariant under a frame transformation (1.3). We then incorporate the spacetime dependence of the fields in order to construct a grand configuration space in Sec. IX. This allows us to construct a fully frame and spacetime-diffeomorphism-invariant pathintegral measure, which we can then be used to quantize the theory in a reparametrization-invariant way. We provide a concise description of the formalism in Sec. X, before discussing our findings in Sec. XI.

\section{COVARIANCE IN SCALAR FIELD THEORIES}

Let us begin by reviewing the construction of the field space for scalar field theories without gravity. Such theories have actions of the form

$$
\begin{aligned}
S & \equiv \int d^{D} x \sqrt{-g} \mathcal{L}, \\
\mathcal{L} & \equiv \frac{1}{2} g^{\mu \nu} k_{A B}(\boldsymbol{\phi}) \partial_{\mu} \phi^{A} \partial_{\nu} \phi^{B}-V(\boldsymbol{\phi}),
\end{aligned}
$$

where $D$ is the dimension of spacetime. In this section, we will take the metric of spacetime $g_{\mu \nu}$ to be fixed and will not consider any redefinitions of the form (1.4). We will relax this assumption in Sec. VI.

As discussed in the Introduction, we could just as easily describe this theory in terms of a different set of fields $\tilde{\boldsymbol{\phi}}$ and the transformation

$$
\phi^{A} \rightarrow \tilde{\phi}^{A}=\tilde{\phi}^{A}(\boldsymbol{\phi})
$$

is just a change of description and should therefore not affect any calculations. In order to make this fact explicit, we will construct a manifold known as the field space [28-33] and treat the fields $\boldsymbol{\phi}$ as coordinates describing that manifold. With such a construction, the transformation (2.2) is simply a diffeomorphism of the field space. We can then write down the theory in a way that is explicitly reparametrization invariant by simply building it out of field-space covariant objects.

The field space is a Riemannian manifold, and so we can equip it with a metric. Such a metric should satisfy the following three properties [39]: 
(1) It should transform as a symmetric rank 2 tensor under (2.2).

(2) It should be determined from the classical action (2.1).

(iii) It should be Euclidean for a canonically normalized theory.

The only quantity that satisfies these conditions is the model function $k_{A B}(\boldsymbol{\phi})$ and so that is what is used in the literature.

In this paper, we want to introduce a new expression for the field-space metric; one that is constructive, rather than relying on the identification of a particular term in Lagrangian. We will thus define the field-space metric to be

$$
G_{A B} \equiv \frac{g_{\mu \nu}}{D} \frac{\partial^{2} \mathcal{L}}{\partial\left(\partial_{\mu} \phi^{A}\right) \partial\left(\partial_{\nu} \phi^{B}\right)},
$$

where $D$ is the number of spacetime dimensions. Notice that for the theory described by (2.1), this new prescription still gives $G_{A B}=k_{A B}$. However, this new prescription is now constructive and can thus be applied to any field theory-even, for example, those with higher derivative terms. ${ }^{1}$ This constructive prescription also ensures that the field-space metric is unique for a given theory.

With the field-space metric thus defined, we can straightforwardly define a connection on the field-space manifold,

$$
\Gamma_{B C}^{A} \equiv \frac{1}{2} G^{A D}\left[\frac{\partial G_{B D}}{\partial \phi^{C}}+\frac{\partial G_{D C}}{\partial \phi^{B}}-\frac{\partial G_{B C}}{\partial \phi^{D}}\right],
$$

where $G^{A B}$ is the inverse of $G_{A B}$. We can also define a fieldspace covariant derivative,

$$
\begin{aligned}
\nabla_{C} X^{A} & \equiv \frac{\partial X^{A}}{\partial \phi^{C}}+\Gamma_{C D}^{A} X^{D}, \\
\nabla_{C} X_{A} & \equiv \frac{\partial X_{A}}{\partial \phi^{C}}-\Gamma_{C A}^{D} X_{D}
\end{aligned}
$$

and so on in the usual manner for higher-rank tensors.

When quantizing the theory, the field-space manifold alone is not sufficient. In the path-integral formalism, we must integrate not just over the fields, but overall configurations of the fields. In order to construct this integral in a covariant manner, we define an infinite-dimensional configuration-space manifold. Each direction on this manifold represents a different configuration of the fields and thus we can describe it using coordinates

$$
\phi^{a} \equiv \phi^{A}\left(x_{A}\right)
$$

\footnotetext{
${ }^{1}$ In the case of a higher derivative theory, (2.3) would lead to a Finslerian metric [42] - one that depends on both the fields and their derivatives. We will not discuss such theories here, but will save them for future work.
}

The lowercase Latin index $a=\left\{A, x_{A}\right\}$ is a continuous index that runs over all points in spacetime in addition to all the scalar fields in the theory, as described in the Introduction.

In order to define a metric for the configuration space, we need to add one more property to the list above. The configuration-space metric should be ultralocal, i.e., it should be proportional to a Dirac delta function only and contains no derivatives of the fields. We therefore define the configuration-space metric as

$$
\begin{aligned}
\mathcal{G}_{a b} & \equiv \frac{g_{\mu \nu}}{D} \frac{\delta^{2} S}{\delta\left(\partial_{\mu} \phi^{a}\right) \delta\left(\partial_{\nu} \phi^{b}\right)} \\
& =G_{A B} \delta^{(D)}\left(x_{A}-x_{B}\right) .
\end{aligned}
$$

Here we have defined the functional derivative with respect to a partial derivative as

$$
\begin{aligned}
& \frac{\delta F\left[\partial_{\mu} \Phi^{A}(x)\right]}{\delta\left(\partial_{\mu} \Phi^{A}(y)\right)} \\
& \quad \equiv \lim _{\epsilon_{\mu}^{A} \rightarrow 0} \frac{F\left[\partial_{\mu} \Phi^{A}(x)+\epsilon_{\mu}^{A} \delta^{(D)}(x-y)\right]-F\left[\partial_{\mu} \Phi^{A}(x)\right]}{\epsilon_{\mu}^{A}},
\end{aligned}
$$

where the Dirac delta function is normalized such that

$$
\int d^{D} x \sqrt{-g} \delta^{(D)}(x)=1
$$

Such a definition allows $\delta^{(D)}(x)$ to be diffeomorphism invariant. We note that with this definition $\delta \Phi^{A} / \delta\left(\partial_{\mu} \Phi^{A}\right)=0$.

The connection on the configuration-space manifold is as follows:

$$
\begin{aligned}
\Gamma_{b c}^{a} & \equiv \frac{1}{2} \mathcal{G}^{a d}\left[\frac{\delta \mathcal{G}_{b d}}{\delta \phi^{c}}+\frac{\delta \mathcal{G}_{d c}}{\delta \phi^{b}}-\frac{\delta \mathcal{G}_{b c}}{\delta \phi^{d}}\right] \\
& =\Gamma_{B C}^{A} \delta^{(D)}\left(x_{A}-x_{B}\right) \delta^{(D)}\left(x_{A}-x_{C}\right),
\end{aligned}
$$

and thus the configuration-space covariant functional derivative is

$$
\begin{aligned}
& \nabla_{c} X^{a} \equiv \frac{\delta X^{a}}{\delta \phi^{c}}+\Gamma_{c d}^{a} X^{d}, \\
& \nabla_{c} X_{a} \equiv \frac{\delta X_{a}}{\delta \phi^{c}}-\Gamma_{c a}^{d} X_{d},
\end{aligned}
$$

similar to (2.5).

With the configuration-space manifold defined, it is straightforward to write theories in a manifestly reparametrization-invariant way. We simply need to build our theory out of configuration-space tensors and ensure that all indices are fully contracted.

It is also easy to identify quantities that are not reparametrization invariant. Two examples of noninvariant 
objects are the quantum effective action and Feynman diagrams, as we shall show in the following sections.

\section{NONCOVARIANCE OF THE ORDINARY EFFECTIVE ACTION}

The ordinary effective action formalism [35-38] fundamentally stems from the one-particle irreducible (1PI) approach in QFT. Through its application, it is possible to define an action that inherently incorporates all quantum effects beyond tree level, in principle allowing us to study radiative corrections nonperturbatively.

The starting point for the derivation of the effective action is the generating functional for 1PI diagrams,

$$
\begin{aligned}
Z[\boldsymbol{J}] & \equiv \exp \left(\frac{i}{\hbar} W[\boldsymbol{J}]\right) \\
& =\int[\mathcal{D} \boldsymbol{\phi}] \mathcal{M}[\boldsymbol{\phi}] \exp \left[\frac{i}{\hbar} S[\boldsymbol{\phi}]+J_{a} \boldsymbol{\phi}^{a}\right],
\end{aligned}
$$

defined in the presence of an external source field $J_{a} \equiv$ $J_{A}\left(x_{A}\right)$ (also collectively denoted as $\boldsymbol{J}$ ). Here the functional integral element is $[\mathcal{D} \boldsymbol{\phi}] \equiv \prod_{x, A} d \phi^{A}(x)$, and $\mathcal{M}[\boldsymbol{\phi}]$ is the measure of the configuration space for the quantum fields $\phi^{a}$. We have also reintroduced the reduced Planck constant $\hbar$ as a means of keeping track different orders of quantum loops. The generating functional is reminiscent of the partition function in statistical mechanics, which is a weighted sum of Boltzmann factors over the different microstates of the system. In a similar vein, the generating functional is defined as a weighted integral over all possible configurations of the quantum fields $\phi^{a}$ of the system.

From the generating functional, it is possible to arrive at the effective action via the Legendre transformation,

$$
\Gamma[\boldsymbol{\varphi}]=W[\boldsymbol{J}]+i \hbar J_{a} \varphi^{a},
$$

where the $\varphi^{a}$ (collectively denoted as $\varphi$ ) are the mean fields and $J_{a}=J_{a}[\boldsymbol{\varphi}]$ is considered to be a functional of $\boldsymbol{\varphi}$. In the presence of the source terms $J_{a}$, the mean fields and the sources are related by

$$
\varphi^{a}=-i \hbar \frac{\delta W[\boldsymbol{J}]}{\delta J_{a}}, \quad J_{a}=-\frac{i}{\hbar} \frac{\delta \Gamma[\boldsymbol{\varphi}]}{\delta \varphi^{a}} .
$$

The usefulness of the effective action is thus that extremizing it generates the quantum-corrected equations of motion.

Already at this point, it is possible to observe that this construction lacks covariance. Since $\varphi^{a}$ is not a configuration-space vector, $J_{a} \varphi^{a}$ is a frame-dependent expression and thus all three equations (3.1)-(3.3) are sensitive to the way in which we parametrize the fields in our theory. This is a major drawback for this approach and once we shall return to, but for now, let us proceed in order to illustrate how the ordinary effective action is usually derived and pave the way for the derivation of the covariant expression.

The effective action $\Gamma[\boldsymbol{\varphi}]$ satisfies the following implicit functional integro-differential equation:

$$
\begin{aligned}
& \exp \left(\frac{i}{\hbar} \Gamma[\boldsymbol{\varphi}]\right) \\
& =\int[\mathcal{D} \boldsymbol{\phi}] \mathcal{M}[\boldsymbol{\phi}] \exp \left\{\frac{i}{\hbar}\left[S[\boldsymbol{\phi}]+\frac{\delta \Gamma[\boldsymbol{\varphi}]}{\delta \varphi^{a}}\left(\varphi^{a}-\boldsymbol{\phi}^{a}\right)\right]\right\} .
\end{aligned}
$$

Equation (3.4) may be derived by substituting (3.2) and (3.3) in (3.1). Evidently, solving (3.4) exactly is prohibitively hard. Fortunately, it is possible to solve for $\Gamma[\boldsymbol{\varphi}]$ in a perturbative loopwise expansion with the help of the background field method [43], where we split the quantum field $\phi^{a}$ into a background component, which we treat classically, and a quantum perturbation. Similarly, we expand $\Gamma[\boldsymbol{\varphi}]=S_{0}[\boldsymbol{\varphi}]+\hbar \Gamma^{(1)}[\boldsymbol{\varphi}]+\hbar^{2} \Gamma^{(2)}[\boldsymbol{\varphi}]+\cdots$. At each loop order, the path integral can be evaluated explicitly. In detail, at one- and two-loop order, we have

$$
\begin{gathered}
\Gamma^{(1)}[\boldsymbol{\varphi}]=i \ln \mathcal{M}[\boldsymbol{\varphi}]-\frac{i}{2} \ln \operatorname{det} S_{, a b}[\boldsymbol{\varphi}], \\
\Gamma^{(2)}[\boldsymbol{\varphi}]=\frac{1}{8} \Delta^{a b} \Delta^{c d} S_{, a b c d}-\frac{1}{12} \Delta^{a b} \Delta^{c d} \Delta^{e f} S_{, a c e} S_{, b d f}
\end{gathered}
$$

where a comma ${ }_{, a} \equiv \delta / \delta \phi^{a}$ indicates a functional derivative with respect to the field $\phi^{a}$ and

$$
\Delta^{a b} \equiv\left(\frac{\delta^{2} S}{\delta \phi^{a} \delta \phi^{b}}\right)^{-1}
$$

is the propagator.

As we shall explore in detail in Sec. V, (3.6) can be represented graphically by the Feynman diagrams

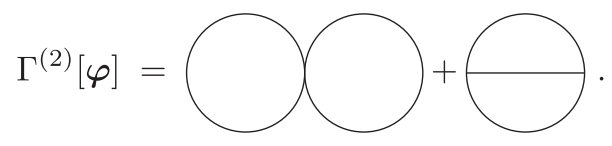

Note that $\Gamma^{(2)}[\boldsymbol{\varphi}]$ contains only 1PI graphs. Other possible one-particle reducible diagrams, such as

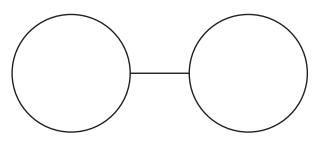

evaluate to zero and so do not contribute to the final expression (3.8).

For our theory to be fully reparametrization invariant, we require that the effective action be a scalar under reparametrizations of the mean fields

$$
\varphi^{a} \rightarrow \tilde{\varphi}^{a}=\tilde{\varphi}^{a}(\varphi)
$$


We saw above that the explicit dependence of the generating functional on the fields $\varphi^{a}$ spoils the covariance, and as a result, such a transformation will not leave (3.5) and (3.6) invariant. This occurs because the difference $\varphi^{a}-\phi^{a}$ does not transform as a vector in configuration space, spoiling the covariance of the term $\frac{\delta \Gamma[\varphi]}{\delta \varphi^{a}}\left(\varphi^{a}-\phi^{a}\right)$ in (3.4). Similarly, the presence of ordinary functional derivatives in (3.5) and (3.6) induces extra terms in the expression for $\Gamma^{(1)}$ and $\Gamma^{(2)}$, which means that the expression for the effective action is not a configuration-space scalar. The parametrization dependence of the effective action can be seen explicitly in Appendix A.

\section{VILKOVISKY AND DEWITT'S SOLUTION: THE COVARIANT EFFECTIVE ACTION}

The VDW effective action formalism [29,39,40,44-47] was developed in order to address the problems of noncovariance of the ordinary effective action that were outlined in the previous section. Unlike the conventional approach, this formalism does not unduly privilege a particular frame. In this section, we review the key results of the VDW formalism.

As noted in Sec. III, the noninvariance of the ordinary effective action stems from the term $\frac{\delta \Gamma}{\delta \varphi^{a}}\left(\phi^{a}-\varphi^{a}\right)$ in (3.4), which is not a configuration-space scalar. Vilkovisky's proposal [39] was therefore to replace the difference $\phi^{a}-\varphi^{a}$ with a two-point quantity $\Sigma^{a}[\boldsymbol{\varphi}, \boldsymbol{\phi}]$ that transforms as a vector with respect to the mean field $\varphi$, a scalar with respect to the quantum field $\boldsymbol{\phi}$ and satisfies $\Sigma^{a}[\boldsymbol{\phi}, \boldsymbol{\phi}]=0$. Making this replacement in (3.4) gives

$$
\begin{aligned}
& \exp \left(\frac{i}{\hbar} \Gamma[\boldsymbol{\varphi}]\right) \\
& =\int[\mathcal{D} \boldsymbol{\phi}] \mathcal{M}[\boldsymbol{\phi}] \exp \left\{\frac{i}{\hbar}\left[S[\boldsymbol{\phi}]+\frac{\delta \Gamma[\boldsymbol{\varphi}]}{\delta \varphi^{a}} \Sigma^{a}[\boldsymbol{\varphi}, \boldsymbol{\phi}]\right]\right\} .
\end{aligned}
$$

There are no frame-dependent terms in (4.1) and therefore this newly defined action is fully frame invariant.

Vilkovisky's original proposal was to use $\Sigma^{a}[\boldsymbol{\varphi}, \boldsymbol{\phi}]=$ $\sigma^{a}[\boldsymbol{\varphi}, \boldsymbol{\phi}]$, where $\sigma^{a}[\boldsymbol{\varphi}, \boldsymbol{\phi}]$ is the tangent vector to the geodesic connecting $\varphi$ and $\phi$ evaluated at $\varphi$. The affinely normalized tangent vector can be found by solving

$$
\sigma^{b}[\boldsymbol{\varphi}, \boldsymbol{\phi}] \nabla_{b} \sigma^{a}[\boldsymbol{\varphi}, \boldsymbol{\phi}]=\sigma^{a}[\boldsymbol{\varphi}, \boldsymbol{\phi}],
$$

along with the boundary conditions

$$
\begin{aligned}
\left.\sigma^{a}[\boldsymbol{\varphi}, \boldsymbol{\phi}]\right|_{\boldsymbol{\varphi = \phi}} & =0, \\
\left.\nabla_{b} \sigma^{a}[\boldsymbol{\varphi}, \boldsymbol{\phi}]\right|_{\boldsymbol{\varphi = \phi}} & =\delta_{B}^{A} \delta^{(D)}\left(x_{A}-x_{B}\right) \equiv \delta_{b}^{a},
\end{aligned}
$$

where $\nabla_{a}$ is the covariant derivative as defined in (2.11) and is taken to act on the first argument $\varphi$. It is possible to expand $\sigma^{a}[\boldsymbol{\varphi}, \boldsymbol{\phi}]$ in terms of the configuration-space connection $\Gamma_{b c}^{a}[\boldsymbol{\varphi}]$ as

$$
\begin{aligned}
-\sigma^{a}[\boldsymbol{\varphi}, \boldsymbol{\phi}]= & -\left(\varphi^{a}-\phi^{a}\right) \\
& +\frac{1}{2} \Gamma_{b c}^{a}[\boldsymbol{\varphi}]\left(\varphi^{b}-\phi^{b}\right)\left(\varphi^{c}-\phi^{c}\right)+\cdots
\end{aligned}
$$

However, $\sigma^{a}$ is not the only possible choice of two-point quantity that satisfies the required properties to make the action frame invariant. In fact, any superposition of tangent vectors

$$
\Sigma^{a}[\boldsymbol{\varphi}, \boldsymbol{\phi}]=\left(C^{-1}[\boldsymbol{\varphi}]\right)_{b}^{a} \sigma^{b}[\boldsymbol{\varphi}, \boldsymbol{\phi}]
$$

will do. We therefore need to introduce another requirement to fix the matrix $C_{b}^{a}$.

For theories with a flat configuration space, we can always go to a frame in which the metric is Euclidean and all the connections vanish. In such a frame, there should be no nontrivial field-space effects and thus the VDW effective action should agree with the ordinary effective action calculated in the previous section. It can be shown [48] that this requirement forces us to choose $C_{b}^{a}=\delta_{b}^{a}$ for such theories. However, for theories with nonzero configurationspace curvature, no such frame exists and so a different condition is required to fix $C_{b}^{a}$.

The choice made by DeWitt [40] is the condition of vanishing tadpoles

$$
\left\langle\Sigma^{a}[\boldsymbol{\varphi}, \boldsymbol{\phi}]\right\rangle_{\Sigma}=0
$$

where the expectation value is defined as

$$
\begin{aligned}
\langle F[\boldsymbol{\varphi}, \boldsymbol{\phi}]\rangle_{\Sigma}= & \exp \left(-\frac{i}{\hbar} \Gamma[\boldsymbol{\varphi}]\right) \int[\mathcal{D} \boldsymbol{\phi}] \mathcal{M}[\boldsymbol{\phi}] F[\boldsymbol{\varphi}, \boldsymbol{\phi}] \\
& \times \exp \left\{\frac{i}{\hbar}\left[S[\boldsymbol{\phi}]+\frac{\delta \Gamma[\boldsymbol{\varphi}]}{\delta \varphi^{a}} \Sigma^{a}[\boldsymbol{\varphi}, \boldsymbol{\phi}]\right]\right\} .
\end{aligned}
$$

This choice was made for two main reasons. First, it allows the effective action to be calculated perturbatively as a sum of 1PI Feynman diagrams [49]. Second, when the formalism is extended to gauge theories, (4.6) is vital in ensuring that the resulting effective action is independent of the choice of gauge-fixing conditions $[44,48]$.

In order to satisfy (4.6), we find that we require [40]

$$
\begin{aligned}
C_{b}^{a}[\boldsymbol{\varphi}] & =\left\langle\nabla_{b} \sigma^{a}[\boldsymbol{\varphi}, \boldsymbol{\phi}]\right\rangle_{\Sigma} \\
& =\left\langle\delta_{b}^{a}-\frac{1}{3} R_{c b d}^{a}[\boldsymbol{\varphi}] \sigma^{c}[\boldsymbol{\varphi}, \boldsymbol{\phi}] \sigma^{d}[\boldsymbol{\varphi}, \boldsymbol{\phi}]+\ldots\right\rangle_{\Sigma} .
\end{aligned}
$$

Here $R^{a}{ }_{c b d}$ is the Riemann tensor of the configurationspace manifold. Notice that the Riemann tensor for a flat manifold is $R_{c b d}^{a}=0$, and thus we recover Vilkovisky's original proposal in this case. 
We can use the background field method to expand (4.1) perturbatively, exactly as we did for the ordinary effective action (3.4). This gives us the following equations for the one- and two-loop corrections to the VDW effective action [48]:

$$
\begin{gathered}
\Gamma^{(1)}[\boldsymbol{\varphi}]=-\frac{i}{2} \ln \operatorname{det} \nabla^{a} \nabla_{b} S \\
\Gamma^{(2)}[\boldsymbol{\varphi}]=\frac{1}{8} \Delta^{a b} \Delta^{c d} \nabla_{(a} \nabla_{b} \nabla_{c} \nabla_{d)} S \\
-\frac{1}{12} \Delta^{a b} \Delta^{c d} \Delta^{e f}\left(\nabla_{(a} \nabla_{c} \nabla_{e)} S\right)\left(\nabla_{(b} \nabla_{d} \nabla_{f)} S\right)
\end{gathered}
$$

where $\Delta^{a b}=\left(\nabla_{a} \nabla_{b} S\right)^{-1}$ is the covariant propagator and the parentheses $(\ldots)$ denote symmetrization with respect to the indices enclosed. Notice that $\Gamma^{(1)}$ and $\Gamma^{(2)}$ are both now invariant under a frame transformation (3.10) as expected.

It was noted in $[48,49]$ that the VDW effective action defined in (4.1) does not generate the covariant correlation functions of $\varphi$ in its current form. In order to achieve this, we must instead define

$$
\begin{aligned}
& \exp \left(\frac{i}{\hbar} \tilde{\Gamma}\left[\boldsymbol{\varphi}, \boldsymbol{\varphi}_{0}\right]\right) \\
& =\int[\mathcal{D} \boldsymbol{\phi}] \mathcal{M}[\boldsymbol{\phi}] \\
& \quad \times \exp \left\{\frac{i}{\hbar}\left[S[\boldsymbol{\phi}]+\frac{\delta \tilde{\Gamma}\left[\boldsymbol{\varphi}, \boldsymbol{\varphi}_{0}\right]}{\delta \varphi^{a}}\left(\Sigma^{a}\left[\boldsymbol{\varphi}_{0}, \boldsymbol{\phi}\right]-\Sigma^{a}\left[\boldsymbol{\varphi}_{0}, \boldsymbol{\varphi}\right]\right)\right]\right\}
\end{aligned}
$$

where $\varphi_{0}$ is an arbitrary base point.

The effective action in (4.11) depends explicitly on the base point $\varphi_{0}$, and so one may question its uniqueness. However, as shown in [49], this explicit dependence of $\tilde{\Gamma}\left[\boldsymbol{\varphi}, \boldsymbol{\varphi}_{0}\right]$ on $\boldsymbol{\varphi}_{0}$ gets cancelled against the implicit dependence of $\boldsymbol{\varphi}=\boldsymbol{\varphi}\left(\boldsymbol{\varphi}_{0}\right)$ evaluated at the same base point, i.e.,

$$
\frac{\delta}{\delta \varphi_{0}^{a}} \tilde{\Gamma}\left[\boldsymbol{\varphi}\left(\boldsymbol{\varphi}_{0}\right), \boldsymbol{\varphi}_{0}\right]=0
$$

Hence, $\tilde{\Gamma}\left[\boldsymbol{\varphi}\left(\boldsymbol{\varphi}_{0}\right), \boldsymbol{\varphi}_{0}\right]$ is independent of $\boldsymbol{\varphi}_{0}$.

As a consequence, one may consider a simplified scheme, in which $\varphi_{0}$ is identified with $\boldsymbol{\varphi}$, such that $\Sigma^{a}\left[\boldsymbol{\varphi}_{0}, \boldsymbol{\varphi}\right]$ vanishes on the rhs of (4.11). In this simplified scheme, we recover the VDW effective action, where $\Gamma[\boldsymbol{\varphi}]=\tilde{\Gamma}[\boldsymbol{\varphi}, \boldsymbol{\varphi}]$. However, when calculating higher order $n$-point correlation functions, the above identification of $\varphi_{0}$ with $\varphi$ must be made only after any covariant differentiation with respect to $\varphi$ in order to avoid introducing spurious terms.
For brevity, we shall only present the VDW effective action (4.1) in this paper. Nevertheless, it is straightforward to introduce a base point and generalize to (4.11) by simply making the replacement,

$$
\Sigma^{a}[\boldsymbol{\varphi}, \boldsymbol{\phi}] \rightarrow \Sigma^{a}\left[\boldsymbol{\varphi}_{0}, \boldsymbol{\phi}\right]-\Sigma^{a}\left[\boldsymbol{\varphi}_{0}, \boldsymbol{\varphi}\right] .
$$

It is important to note that, on shell, we have $\frac{\delta \Gamma}{\delta \varphi^{a}}=0$, in which case the expressions for the ordinary effective action (3.4) and the VDW effective action (4.1) are identical. Thus, we are guaranteed to get the same results for on-shell observables regardless of whether we use the ordinary effective action (3.4) or the VDW effective action (4.1). This also means that any parametrization dependence that arises when using the ordinary effective action must vanish when the calculations are performed on shell. We show some examples of this in Appendix A.

The fact that the VDW formalism remains covariant off shell is important for a few reasons, even if off-shell quantities will never appear in observables. First, from a geometric point of view, we expect covariance to be satisfied for the entirety of the configuration space, not just the geodesics. The ordinary approach is parametrizationindependent only for a severely restricted subspace (the onshell region), and so the VDW approach is required to restore covariance for the whole configuration space. Second, offshell formulations of QFTs have many important applications, such as in supersymmetry [50] and the analysis of quantum anomalies [51]. Finally, inflationary observables are often computed in the slow-roll approximation [52]. Such an approximation forces us to perform calculations in the off-shell regime.

\section{COVARIANT FEYNMAN RULES}

In the previous section, we showed how the quantum effective action can be constructed in a fully covariant way. However, in practice, radiative corrections are often calculated perturbatively with the help of Feynman diagrams. As we will show in this section, usual Feynman diagrams are also inherently noncovariant. As such, their form depends on the parametrization used to calculate them. They should therefore be replaced with an alternative, fully covariant method of calculating Feynman rules. Such a covariant expansion was first developed by Honerkamp [53-55] in the context of chiral pion theories, but can be readily extended to any scalar field theory as shown below. In this section, we provide an explicit derivation of the formalism before applying it to specific examples in Appendices A, B, and $\mathrm{C}$.

We first review how ordinary Feynman diagrams may be employed to calculate correlation functions (as well as S-matrix elements through the Lehmann-SymanzikZimmermann reduction formula [56]). The derivation can be found in most textbooks on QFT (see, e.g., [57]), but our treatment here most closely follows $[58,59]$. 
In the path-integral formulation of QFT, a correlation function in the presence of a source $\boldsymbol{J}$ is given by

$$
\left\langle\boldsymbol{\phi}^{a} \boldsymbol{\phi}^{b} \ldots, \boldsymbol{J}\right\rangle=\frac{\int[\mathcal{D} \boldsymbol{\phi}] \mathcal{M}[\boldsymbol{\phi}]\left(\boldsymbol{\phi}^{a} \boldsymbol{\phi}^{b} \ldots\right) e^{\frac{i}{\hbar} S\left[\boldsymbol{\phi}_{\mathbf{0}}+\boldsymbol{\phi}\right]+J_{a} \phi^{a}}}{\int[\mathcal{D} \boldsymbol{\phi}] \mathcal{M}[\boldsymbol{\phi}] e^{\frac{i}{\hbar} S\left[\boldsymbol{\phi}_{\mathbf{0}}+\boldsymbol{\phi}\right]+J_{a} \phi^{a}}},
$$

where $\phi_{0}$ is an arbitrary point around which we quantizeusually taken to be the classical vacuum. This can be calculated using the generating functional $Z[\boldsymbol{J}]$ defined in (3.1). In terms of this generating functional, the correlation function becomes

$$
\left\langle\phi^{a} \phi^{b} \ldots, \boldsymbol{J}\right\rangle=\frac{1}{Z[\boldsymbol{J}]}\left(\frac{\delta}{\delta J_{a}} \frac{\delta}{\delta J_{b}} \ldots\right) Z[\boldsymbol{J}] .
$$

In order to perform perturbative calculations, we use a Taylor series expansion of the action

$$
S\left[\boldsymbol{\phi}_{\mathbf{0}}+\boldsymbol{\phi}\right]=\sum_{N} S_{a_{1} \ldots a_{N}}^{(N)} \phi^{a_{1}} \ldots \phi^{a_{N}}
$$

where

$$
S_{a_{1} \ldots a_{N}}^{(N)}=\left.\frac{1}{N !} \frac{\delta^{N} S}{\delta \phi^{a_{1}} \ldots \delta \phi^{a_{N}}}\right|_{\phi_{0}} .
$$

The constant term $S^{(0)}$ gives factors which cancel out in (5.1) and therefore we will therefore ignore it. We will also take $\phi_{0}$ to be the classical vacuum so that we have $S_{a}^{(1)}=0$. The lowest order nontrivial term in our expansion is therefore

$$
S\left[\boldsymbol{\phi}_{\mathbf{0}}+\boldsymbol{\phi}\right] \approx S_{a b}^{(2)} \phi^{a} \phi^{b}
$$

about which we shall expand the generating functional $Z[\boldsymbol{J}]$.

We must also Taylor expand the path-integral measure. Using Vilkovisky's suggestion of $\mathcal{M}[\boldsymbol{\phi}]=\sqrt{\operatorname{det} \mathcal{G}_{a b}}$, we find [29]

$$
\begin{aligned}
\mathcal{M}\left[\boldsymbol{\varphi}_{\mathbf{0}}+\boldsymbol{\varphi}\right]= & 1+\delta^{(D)}(0) \int d^{D} x \sqrt{-g} \operatorname{Tr} \ln G_{A B}[\boldsymbol{\phi}(x)] \\
& +\ldots
\end{aligned}
$$

From this expansion, we see that all nontrivial effects of the measure are proportional to $\delta^{(D)}(0)$. This is a simple divergence equal to the total volume of the spacetime manifold and, as such, will be removed by our regularization procedure. Therefore, the functional form of the measure will have no impact on perturbative results and so we can set $\mathcal{M}=1$.

With this knowledge, and the expansion given in (5.4), we can write

$$
\begin{aligned}
Z[\boldsymbol{J}]= & \exp \left(\frac{i}{\hbar} \sum_{N>2} S_{a_{1} \ldots a_{N}}^{(N)} \frac{\delta}{\delta J_{a_{1}}} \ldots \frac{\delta}{\delta J_{a_{N}}}\right) \\
& \times \int[\mathcal{D} \boldsymbol{\phi}] e^{\frac{i}{\hbar^{2}} S_{a b}^{(2)} \phi^{a} \phi^{b}+J_{a} \phi^{a}} .
\end{aligned}
$$

The functional integral is now Gaussian and so can be calculated explicitly. The result is

$$
\begin{aligned}
Z[\boldsymbol{J}]= & \mathcal{N} \exp \left(\frac{i}{\hbar} \sum_{N>2} S_{a_{1} \ldots a_{N}}^{(N)} \frac{\delta}{\delta J_{a_{1}}} \ldots \frac{\delta}{\delta J_{a_{N}}}\right) \\
& \times \exp \left(-i \hbar J_{a} \Delta^{a b} J_{b}\right),
\end{aligned}
$$

where $\Delta^{a b}$ is the inverse of $S_{a b}^{(2)}$, often known as the propagator, and $\mathcal{N}$ is an irrelevant normalization factor.

Expanding out the two exponentials, we see that the correlation function (5.2) is

$$
\begin{aligned}
\left\langle\phi^{a} \phi^{b} \ldots, \boldsymbol{J}\right\rangle= & \frac{\mathcal{N}}{Z[\boldsymbol{J}]}\left(\frac{\delta}{\delta J_{a}} \frac{\delta}{\delta J_{b}} \ldots\right) \\
& \times \prod_{N>2}\left[\sum_{V_{N}=0}^{\infty} \frac{1}{V_{N} !}\left(\frac{i}{\hbar} S_{a_{1} \ldots a_{N}}^{(N)} \frac{\delta}{\delta J_{a_{1}}} \ldots \frac{\delta}{\delta J_{a_{N}}}\right)^{V_{N}}\right] \\
& \times \sum_{P=0}^{\infty} \frac{1}{P !}\left(-i \hbar J_{c} \Delta^{c d} J_{d}\right)^{P} .
\end{aligned}
$$

Feynman diagrams [60] are a beautiful graphical way to keep track of the nonzero terms in (5.9). If we represent each propagator by a line,

$$
a \bullet b=\Delta^{a b}
$$

and each term of the expansion (5.3) with a vertex,

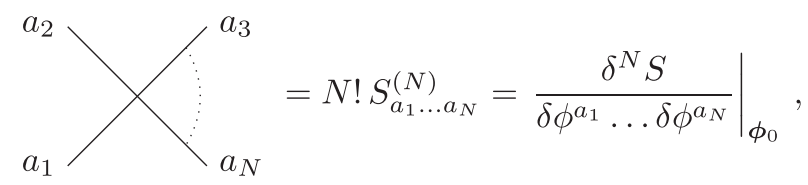

then each term in (5.9) can be expressed as a diagram with $P$ propagators and $V_{N}$ vertices of order $N$. Calculating the correlation function then simply amounts to summing up all possible diagrams with the correct number of external legs. Finally, it can easily be shown that the prefactor $\frac{\mathcal{N}}{Z[J]}$ on the rhs of (5.9) has the effect of removing all diagrams that are not fully connected.

The above derivation is very elegant and has been used extensively in QFT calculations. However, it is not reparametrization invariant. This is because, as we have seen, the quantity $\phi^{a}$ is not a configuration-space vector. Therefore, it will not transform in a covariant manner and cannot be contracted to form reparametrization-invariant quantities. 
This means that the individual terms on the rhs of (5.3) will change under a field redefinition. Although the full sum will remain invariant (since the lhs is a configurationspace scalar), the individual terms will mix into each other and hence any finite truncation of the sum will not be invariant. Moreover, the term $J_{a} \phi^{a}$ in (3.1), as well as the definition of the correlation function (5.1), is not field covariant. As such, their form is dependent on our choice of parametrization. Some examples of the parametrization dependence of ordinary Feynman calculations are shown in Appendix A.

It is therefore clear that a new, covariant approach to Feynman diagrams is required if we are to calculate quantum corrections in a fully covariant manner. The simplest way to achieve such invariance is to replace the coordinate $\phi^{a}$ with a configuration-space vector, much like we did in Sec. IV. However, in contrast to the previous section, we will employ Vilkovisky's original choice and choose it to be the tangent vector in configuration space $\sigma^{a}\left[\boldsymbol{\phi}_{\mathbf{0}}, \boldsymbol{\phi}_{\mathbf{0}}+\boldsymbol{\phi}\right]$. We shall therefore calculate the covariant correlation functions

$$
\left\langle\boldsymbol{\sigma}^{a} \sigma^{b} \ldots, \boldsymbol{J}\right\rangle_{\sigma}=\frac{\int[\mathcal{D} \boldsymbol{\sigma}]\left(\boldsymbol{\sigma}^{a} \sigma^{b} \ldots\right) e^{\frac{i}{h} S\left[\boldsymbol{\phi}_{\mathbf{0}}+\boldsymbol{\phi}\right]+J_{a} \sigma^{a}}}{\int[\mathcal{D} \boldsymbol{\sigma}] e^{\frac{i}{S} S\left[\boldsymbol{\phi}_{\mathbf{0}}+\boldsymbol{\phi}\right]+J_{a} \sigma^{a}}},
$$

where the suppressed arguments of $\sigma^{a}$ are $\left[\boldsymbol{\phi}_{0}, \boldsymbol{\phi}_{0}+\boldsymbol{\phi}\right]$ in all cases. Notice that $[\mathcal{D} \boldsymbol{\phi}] \mathcal{M}[\boldsymbol{\phi}]=[\mathcal{D} \boldsymbol{\sigma}]$ and thus the measure is trivial in this case.

We note that $\sigma^{a}\left[\boldsymbol{\phi}_{\mathbf{0}}, \boldsymbol{\phi}_{\mathbf{0}}+\boldsymbol{\phi}\right]=\phi^{a}+O\left(\phi^{2}\right)$, and therefore the correlation functions (5.1) and (5.12) have the same pole structure. This means that the renormalized on-shell

S-matrix elements

$$
\begin{aligned}
& \prod_{I=1}^{E} \lim _{k_{I}^{2} \rightarrow m_{I}^{2}} \frac{k_{I}^{2}-m_{I}^{2}}{Z_{I}^{\frac{1}{2}}}\left\langle\sigma^{a}\left(k_{1}\right) \sigma^{b}\left(k_{2}\right) \ldots, 0\right\rangle_{\sigma} \\
& \quad=\prod_{I=1}^{E} \lim _{k_{I}^{2} \rightarrow m_{I}^{2}} \frac{k_{I}^{2}-m_{I}^{2}}{Z_{I}^{\frac{1}{2}}}\left\langle\phi^{a}\left(k_{1}\right) \phi^{b}\left(k_{2}\right) \ldots, 0\right\rangle
\end{aligned}
$$

are identical [61]. Here $E$ is the number of external fields in the correlation function, and $m_{I}$ and $Z_{I}$ are the (renormalized) mass and wave function renormalization of particle $I$, respectively. Off shell, however, the correlation functions (5.1) and (5.12) will not be equal in general.

Note that we should continue to use the correlation functions (5.12) to calculate S-matrix elements even in the presence of field-space curvature. The correlation functions of DeWitt's modified two-point quantity $\boldsymbol{\Sigma}$ give only linear combinations of (5.13), as can be seen from (4.5), and therefore should not be used.

Let us modify the definition of the generating function to make it frame invariant,

$$
\tilde{Z}[\boldsymbol{J}]=\int[\mathcal{D} \boldsymbol{\sigma}] e^{\frac{i}{\hbar} S\left[\boldsymbol{\phi}_{0}+\boldsymbol{\phi}\right]+J_{a} \sigma^{a}\left[\boldsymbol{\phi}_{0}, \boldsymbol{\phi}_{0}+\boldsymbol{\phi}\right]}
$$

We then find that the correlation functions are given by

$$
\left\langle\sigma^{a} \sigma^{b} \ldots, \boldsymbol{J}\right\rangle_{\sigma}=\frac{1}{\tilde{Z}[\boldsymbol{J}]}\left(\frac{\delta}{\delta J_{a}} \frac{\delta}{\delta J_{b}} \ldots\right) \tilde{Z}[\boldsymbol{J}] .
$$

Finally, we consider an alternative, but equivalent, covariant expansion of the action [39], given by

$S\left[\boldsymbol{\phi}_{\mathbf{0}}+\boldsymbol{\phi}\right]=\sum_{N} \tilde{S}_{a_{1} \ldots a_{n}}^{(N)} \sigma^{a_{1}}\left[\boldsymbol{\phi}_{\mathbf{0}}, \boldsymbol{\phi}_{\mathbf{0}}+\boldsymbol{\phi}\right] \ldots \sigma^{a_{n}}\left[\boldsymbol{\phi}_{\mathbf{0}}, \boldsymbol{\phi}_{\mathbf{0}}+\boldsymbol{\phi}\right]$,

where

$$
\tilde{S}_{a_{1} \ldots a_{n}}^{(N)}=\left.\frac{1}{N !} \nabla_{\left(a_{1}\right.} \ldots \nabla_{\left.a_{n}\right)} S\right|_{\phi_{0}}
$$

and $(\cdots)$ refers to symmetrization over all indices. Now, since $\sigma^{a}$ is a genuine field-space vector, all $\tilde{S}^{(N)}$ are fully covariant field-space tensors, and every term in (5.16) is independently reparametrization invariant.

We can repeat the same derivation as above to calculate the correlation functions graphically by using Feynman diagrams. Now, however, the Feynman rules must be calculated covariantly with the propagator being given by

$$
a \bullet b=\left(\left.\nabla_{a} \nabla_{b} S\right|_{\phi_{0}}\right)^{-1},
$$

and the vertex factors given by

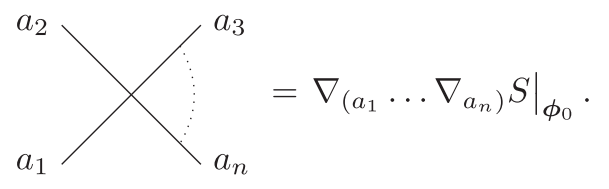

Notice that the Feynman rule is symmetrized over its indices. This is because only the symmetrized version of (5.17) appears in (5.16). For (5.11), this symmetrization had no effect since the ordinary functional derivative is already symmetric. However, for theories with curved field space, covariant functional derivatives do not commute and as a result, this symmetrization is vital in fixing the order of differentiation.

In Appendices A, B, and C, we perform some explicit calculations using the covariant Feynman approach, demonstrating its relation to results obtained in the ordinary approach.

\section{THE GEOMETRIC STRUCTURE OF GRAVITY}

So far, we have treated gravity as a background and have not considered the metric $g_{\mu \nu}$ to be a field. However, the Vilkovisky-DeWitt covariant approach explored in the 
previous sections can be readily applied to tensor fields $[17,39,62]$. We therefore promote $g_{\mu \nu}$ to become a fully dynamical field. Doing so will lead us to the construction of the field space for gravitational theories. This space is a Riemannian manifold and is distinct from the manifold of spacetime. The goal of this section is to illustrate the geometrical features of gravity as described by general relativity.

We begin by examining the action for general relativity, described by the Einstein-Hilbert action,

$$
S=-\frac{1}{2} \int d^{D} x \sqrt{-g} R
$$

We use the standard definitions

$$
\begin{aligned}
\Gamma_{\mu \nu}^{\alpha} & =\frac{g^{\alpha \beta}}{2}\left(g_{\beta \nu, \mu}+g_{\mu \beta, \nu}-g_{\mu \nu, \beta}\right), \\
R_{\mu \beta \nu}^{\alpha} & =\Gamma_{\nu \mu, \beta}^{\alpha}-\Gamma_{\beta \mu, \nu}^{\alpha}+\Gamma_{\beta \lambda}^{\alpha} \Gamma_{\nu \mu}^{\lambda}-\Gamma_{\nu \lambda}^{\alpha} \Gamma_{\beta \mu}^{\lambda}, \\
R_{\mu \nu} & =R^{\alpha}{ }_{\mu \alpha \nu}, \\
R & =g^{\mu \nu} R_{\mu \nu}
\end{aligned}
$$

for the spacetime Christoffel symbols, Riemann tensor, Ricci tensor, and Ricci scalar, respectively.

This action is, famously, nonrenormalizable [63,64]. Because of this, the construction of a UV-complete quantum theory of gravity has yet to be achieved and remains one of the most important open problems in physics. Performing such a construction is far beyond the scope of this paper and, as such, we make no attempt to solve the issue of nonrenormalizability. However, we believe that the issues identified in this section, in particular the curvature of the field space of GR, will be important to consider in any future worked aimed at solving these problems.

For $D$-dimensional gravity, the $D(D+1) / 2$ degrees of freedom of the field space will be represented by an unordered pair of spacetime indices $(\mu \nu)$. In order to maintain consistency with the position of the indices, we take the fundamental field to be $g^{\mu \nu}$. This means that $\delta g^{\mu \nu}$ is a contravariant vector in field space and $\delta g_{\mu \nu}$ is a covariant vector.

As discussed in Sec. II, the field-space metric can be explicitly calculated from the classical action (6.1) by using (2.3). However, there is a subtlety with gravity, stemming from its gauge freedom. This freedom requires us to add to the action a gauge-fixing term of the form

$$
S_{\mathrm{GF}}=-\frac{\gamma}{2} \int d^{D} x \sqrt{-g} \chi^{\mu} g_{\mu \nu} \chi^{\nu}
$$

Here, $\chi^{\mu}=0$ is the gauge-fixing condition and $\gamma$ is a nonnegative constant. When we apply (2.3) to the sum of (6.1) and (6.3), we get the metric ${ }^{2}$

\footnotetext{
${ }^{2}$ Note that we can also arrive at (6.4) up to an irrelevant normalization simply by enforcing that $G_{(\mu \nu)(\rho \sigma)}$ transforms as a spacetime tensor and is symmetric under $\mu \leftrightarrow \nu$ and $\rho \leftrightarrow \sigma$.
}

$$
G_{(\mu \nu)(\rho \sigma)}=\frac{1}{2}\left(g_{\mu \rho} g_{\sigma \nu}+g_{\mu \sigma} g_{\rho \nu}-\alpha g_{\mu \nu} g_{\rho \sigma}\right),
$$

where $\alpha=\alpha\left(\chi^{\mu}, \gamma\right)$ is a constant that depends on the gaugefixing condition $\chi^{\mu}$ and the constant $\gamma$. For example, in de Donder gauge $g^{\rho \sigma} \Gamma_{\rho \sigma}^{\mu}=0$, we have $\alpha=2-\gamma$.

Because $\chi^{\mu}$ and $\gamma$ are both arbitrary, we need another condition to fix $\alpha$. The condition we choose is

$$
\left(G^{-1}\right)^{(\mu \nu)(\rho \sigma)}=G^{(\mu \nu)(\rho \sigma)} \equiv g^{\alpha \mu} g^{\beta \nu} g^{\kappa \rho} g^{\lambda \sigma} G_{(\alpha \beta)(\kappa \lambda)},
$$

where $\left(G^{-1}\right)^{(\mu \nu)(\rho \sigma)}$ is the inverse metric satisfying

$$
G_{(\mu \nu)(\rho \sigma)}\left(G^{-1}\right)^{(\rho \sigma)(\kappa \lambda)}=\frac{1}{2}\left(\delta_{\rho}^{\mu} \delta_{\sigma}^{\nu}+\delta_{\sigma}^{\mu} \delta_{\rho}^{\nu}\right) .
$$

Mathematically, this condition is useful, since it means that there is no difference between raising $(\mu \nu)$ indices with the spacetime metric or the field-space metric.

The inverse metric can be calculated from (6.6) and is found to be

$$
\left(G^{-1}\right)^{(\mu \nu)(\rho \sigma)}=\frac{1}{2}\left(g^{\mu \rho} g^{\nu \sigma}+g^{\mu \sigma} g^{\rho \nu}-\frac{2 \alpha}{D \alpha-2} g^{\mu \nu} g^{\rho \sigma}\right) .
$$

The solution to $(6.5)$ is therefore ${ }^{3}$

$$
\alpha=\frac{4}{D} .
$$

Thus, in four dimensions, (6.4) reduces to

$G_{(\mu \nu)(\rho \sigma)}=P_{\mu \nu \rho \sigma} \equiv \frac{1}{2}\left(g_{\mu \rho} g_{\sigma \nu}+g_{\mu \sigma} g_{\rho \nu}-g_{\mu \nu} g_{\rho \sigma}\right)$,

where $P_{\mu \nu \rho \sigma}$ is Vilkovisky's metric for gravity, derived in [39] by different considerations.

Note that this differs from the DeWitt metric [67], which imposes a time slicing condition and focuses only on the spatial part of the spacetime metric. In contrast, our calculation considers all components of the spacetime metric equally. This allows the metric (6.9) to transform as a tensor under diffeomorphisms of the full spacetime.

We note that the metric (6.9) can be projected onto the space of gauge orbits if one wants to maintain manifest gauge invariance of the VDW effective action [39]. While gauge dependence of the effective action is an important topic, and indeed was one of the original motivations for Vilkovisky's work, it runs parallel to our objective of frame invariance and has been much studied in the literature $[29,44,46,68,69]$. For simplicity, we shall therefore ignore

\footnotetext{
${ }^{3}$ The solution $\alpha=0$ also allows satisfies this equation. However, we choose to use the solution in (6.8), since it agrees with Vilkovisky's original calculation [39] in $D=4$, as well as other results in the literature $[65,66]$.
} 
this complication in the remainder of the paper. The configuration-space metric (6.9) is good enough to achieve our goal of manifest reparametrization invariance.

We are now equipped to determine the curvature of the field space for gravity. The expressions for the curvature tensors are identical to those for spacetime, but with spacetime indices replaced with field-space indices. Thus, the field-space Christoffel symbols and Riemann tensor are given as

$$
\Gamma_{(\mu \nu)(\rho \sigma)}^{(\alpha \beta)}=\frac{1}{2} P^{\alpha \beta \gamma \delta}\left(\partial_{(\mu \nu)} P_{\gamma \delta \rho \sigma}+\partial_{(\rho \sigma)} P_{\mu \nu \gamma \delta}-\partial_{(\gamma \delta)} P_{\mu \nu \rho \sigma}\right),
$$

$$
\begin{aligned}
\mathfrak{R}^{(\mu \nu)}{ }_{(\alpha \beta)(\rho \sigma)(\gamma \delta)}= & \partial_{(\rho \sigma)} \Gamma_{(\gamma \delta)(\alpha \beta)}^{(\mu \nu)}-\partial_{(\gamma \delta)} \Gamma_{(\rho \sigma)(\alpha \beta)}^{(\mu \nu)} \\
& +\Gamma_{(\rho \sigma)(\kappa \lambda)}^{(\mu \nu)} \Gamma_{(\gamma \delta)(\alpha \beta)}^{(\kappa \lambda)}-\Gamma_{(\gamma \delta)(\kappa \lambda)}^{(\mu \nu)} \Gamma_{(\rho \sigma)(\alpha \beta)}^{(\kappa \lambda)},
\end{aligned}
$$

respectively, where $\partial_{(\mu \nu)} \equiv \partial / \partial g^{\mu \nu}$. Correspondingly, the field-space Ricci tensor and Ricci scalar for gravity are given by

$$
\begin{aligned}
\mathfrak{R}_{(\alpha \beta)(\gamma \delta)} & =\mathfrak{R}^{(\mu \nu)}{ }_{(\alpha \beta)(\mu \nu)(\gamma \delta)}, \\
\mathfrak{R} & =P^{\alpha \beta \gamma \delta} \boldsymbol{R}_{(\alpha \beta)(\gamma \delta)} .
\end{aligned}
$$

To cope with the complexity of this calculation, we employed the symbolic computer algebra system CADABRA2 [70,71]. In this way, we find the following explicit forms for the Riemann tensor $\mathfrak{R}^{(\mu \nu)}{ }_{(\alpha \beta)(\rho \sigma)(\gamma \delta)}$ (shown in Appendix D), the Ricci tensor:

$$
\mathfrak{R}_{(\mu \nu)(\rho \sigma)}=\frac{1}{4} g_{\mu \nu} g_{\rho \sigma}-\frac{D}{8} g_{\mu \rho} g_{\nu \sigma}-\frac{D}{8} g_{\mu \sigma} g_{\nu \rho}
$$

and the Ricci scalar

$$
\mathfrak{R}=\frac{D}{4}-\frac{D^{2}}{8}-\frac{D^{3}}{8} .
$$

These tensors are all nonzero (except when $D=1$, which is expected since one-dimensional curvature is impossible). Therefore, this shows that gravity has a genuinely curved field space. Indeed, we can see from (6.14) that the field space is always negatively curved. It would be interesting to explore whether this negative curvature is the origin for the nonconvergence of the path integral for pure gravity.

\section{THE COSMOLOGICAL FRAME PROBLEM IN SCALAR-TENSOR THEORIES}

After studying scalar field theories and gravitational theories separately, we now wish to combine the methods of the previous sections and look at theories with both scalar fields and gravity. In the following two sections, we will therefore construct a covariant formalism for scalartensor theories with an action of the form (1.1).

However, before we do so, we must address the cosmological frame problem, which stems from a subtlety regarding spacetime-diffeomorphism invariance in scalartensor theories. Diffeomorphism invariance is normally achieved by identifying the graviton field $g_{\mu \nu}$ as the metric of spacetime and thus defining the spacetime line element as

$$
d s^{2}=g_{\mu \nu} d x^{\mu} d x^{\nu}
$$

However, when $g_{\mu \nu}$ is taken to be a dynamical field, the rhs of (7.1) is no longer reparametrization invariant. Indeed, it picks up a conformal factor $\Omega^{2}$ under a conformal transformation (1.4). In contrast, the spacetime line element $d s^{2}$ is a measurable quantity and so must be invariant under reparametrizations of the fields.

Previous authors $[10,17,72]$ have dealt with this (either explicitly or implicitly) by choosing a "preferred frame" in which the frame-dependent relation (7.1) holds. Different choices of this preferred frame lead to different quantum corrections for otherwise identical theories, even when these corrections are calculated on shell.

In order to avoid the cosmological frame problem, we shall use a different, frame invariant, definition of the metric of spacetime. The most general such definition that does not require the introduction of any new spacetime tensors and has no momentum dependence is

$$
\bar{g}_{\mu \nu} \equiv \frac{g_{\mu \nu}}{\ell^{2}(\boldsymbol{\phi}(x))}
$$

where $\bar{g}_{\mu \nu}$ is the metric of spacetime and $\ell$ is a (generally spacetime dependent) length scale. In this paper, we will restrict ourselves to the case where $\ell$ depends on $x$ only through the scalar fields $\boldsymbol{\phi}$ in which case $\ell(\boldsymbol{\phi})$ represents another nonsingular model function in our theory. ${ }^{4}$

Provided that $\ell$ transforms as

$$
\ell \rightarrow \tilde{\ell}=\Omega \ell
$$

under conformal transformations (1.4) and does not transform under scalar field redefinitions (1.5), then $\bar{g}_{\mu \nu}$ is frame invariant. Thus, we may define a spacetime line element

$$
d \bar{s}^{2}=\bar{g}_{\mu \nu} d x^{\mu} d x^{\nu}
$$

which is both frame and diffeomorphism invariant. This line element is also dimensionless, in contrast to the standard definition, and therefore qualifies as an observable according to the Buckingham- $\pi$ theorem [73]. Previous

\footnotetext{
${ }^{4}$ Note that if we do not make this assumption, then $\ell(x)$ would act as a new field in the theory and we would have to quantize it accordingly.
} 
authors $[9,74-76]$ have defined similar frame-invariant line elements, but have assumed a particular form of $\ell$. To the best of our knowledge, we are the first to identify $\ell(\boldsymbol{\phi})$ as a freely selectable model function that must be specified when defining a scalar-tensor theory.

At first glance, it may appear that $\ell$ has no physical meaning. After all, it does not appear anywhere in the classical action (1.1) and so will have no effect on any classical observable. However, as we will show below, $\ell$ does appear in the functional measure of the path integral and therefore the choice of $\ell$ will have an observable impact at the quantum level.

Specifying a particular form of $\ell(\boldsymbol{\phi})$ is mathematically equivalent to specifying a preferred frame in the ordinary approach. The frame in which the metric of spacetime $\bar{g}_{\mu \nu}=g_{\mu \nu}$ is the one in which $\ell(\boldsymbol{\phi})=1$, which we shall refer to as the metric frame. The effects of a nontrivial $\ell(\boldsymbol{\phi})$ are therefore equivalent to the so-called frame discriminant calculated in [17].

However, in our formalism the metric frame is no more preferred than any other and thus we are able to write down a scalar-tensor theory of gravity without ever singling out a particular frame. This distinction, although subtle, is vital in constructing a unique, reparametrization-invariant effective action. In addition, by defining a reparametrizationinvariant spacetime line element (7.4), we can see explicitly how the form of $\ell(\boldsymbol{\phi})$ affects the theory, as we will show in the following sections.

Note that in general the Einstein frame and the metric frame are different, and it is not always possible to choose a frame both with minimal coupling $f(\boldsymbol{\phi})=1$, and with $\ell(\boldsymbol{\phi})=1$. Because of this, the metric $\bar{g}_{\mu \nu}$ will not, in general, obey Einstein's equations even in the Einstein frame. This is to be expected. Einstein's equations are the equations of motion that arise upon varying the action with respect to the gravitational tensor field $g_{\mu \nu}$. It is therefore this tensor field that obeys Einstein's equations (in the Einstein frame), and it is $g_{\mu \nu}$ that will form part of our grand field space.

Let us therefore clarify the difference between $\bar{g}_{\mu \nu}$ and $g_{\mu \nu}$. The metric $\bar{g}_{\mu \nu}$ is the metric of spacetime and therefore appears in the spacetime line element (7.4) as well as in the construction of all spacetime-invariant objects. Conversely, $g_{\mu \nu}$ is a field, on the same footing as the scalar fields $\boldsymbol{\phi}$, and thus appears in all equations of motion as well as in Feynmann diagrams. When solving the equations of motion to calculate the field configuration, it is $g_{\mu \nu}$ that must be calculated and thus Einstein's equations may still be used (provided one works in the Einstein frame).

\section{THE GRAND FIELD SPACE}

In this section, we will construct an augmented fieldspace manifold that incorporates both the scalar fields $\phi^{A}$ and the gravitational tensor field $g^{\mu \nu}[17,77]$. To this end, we shall define the following coordinate chart:

$$
\Phi^{I}=\left(\begin{array}{c}
g^{\mu \nu} \\
\phi^{A}
\end{array}\right)
$$

where $I=\{\mu \nu, A\}$. We call this augmented space the grand field space.

As mentioned in the Introduction, any physical observable should be invariant under reparametrizations of the fields. Such reparametrizations are nothing but diffeomorphisms of the grand field space. In fact, the transformations (1.3) can be reexpressed in this notation as

$$
\Phi^{I} \rightarrow \tilde{\Phi}^{I}(\boldsymbol{\Phi})
$$

We now equip our grand field space with a metric. We wish to define the metric in such a way that both spacetimediffeomorphism invariance and invariance under (8.2) remain manifest. To do so, we first define the invariant Lagrangian

$$
\overline{\mathcal{L}}=\ell^{D} \mathcal{L}
$$

such that

$$
S=\int d^{D} x \sqrt{-g} \mathcal{L}=\int d^{D} x \sqrt{-\bar{g}} \overline{\mathcal{L}} .
$$

This definition allows $\overline{\mathcal{L}}$ to be invariant under both spacetime diffeomorphisms and (8.2). This is in contrast to the standard Lagrangian $\mathcal{L}$, which picks up a conformal factor under the conformal transformation (1.4).

We can now define the metric of the grand field space in a way analogous to (2.3). Explicitly, we have

$$
G_{I J}=\frac{\bar{g}_{\mu \nu}}{D} \frac{\partial^{2} \overline{\mathcal{L}}}{\partial\left(\partial_{\mu} \Phi^{I}\right) \partial\left(\partial_{\nu} \Phi^{J}\right)} .
$$

It is important to note that the effective Planck length $\ell$ is now part of the definition of the field-space metric, since it appears in the definition of $\bar{g}_{\mu \nu}$.

For the scalar-tensor theory described by (1.1) in four dimensions, the field-space metric is

$$
G_{I J}=\ell^{2}\left(\begin{array}{cc}
f P_{\mu \nu \rho \sigma} & -\frac{3}{4} f_{, B} g_{\mu \nu} \\
-\frac{3}{4} f_{, A} g_{\rho \sigma} & k_{A B}
\end{array}\right),
$$

where $P_{\mu \nu \rho \sigma}$ is defined in (6.9).

We note that, as discussed in Sec. VI, the metric (8.6) does not follow directly from (8.5), unless the gauge-fixing term takes the specific form discussed in Sec. VI [c.f. (6.8)]. However, previous works $[29,44,46,48,68,69]$ have shown that one can define a projected field-space metric from $G_{I J}$ in (8.6), such that the resulting VDW effective action is independent of the gauge-fixing condition. One can therefore use the techniques developed in these works in order 
construct this projected field-space metric (8.6) in a unique way.

This metric can be used to define a frame-invariant fieldspace line element, given by

$$
d \sigma^{2}=G_{I J} d \Phi^{I} d \Phi^{J}
$$

By construction, this line element is both spacetime-diffeomorphism invariant and frame invariant. We can also define the connection on the grand field space as

$$
\Gamma_{J K}^{I}=\frac{1}{2} G^{I L}\left[\partial_{J} G_{L K}+\partial_{K} G_{J L}-\partial_{L} G_{J K}\right],
$$

where $\partial_{I} \equiv \partial / \partial \Phi^{I}$. The form of the connection can then be used to construct the field-space covariant derivative

$$
\begin{aligned}
& \nabla_{J} X^{I}=\frac{\partial X^{I}}{\partial \Phi^{J}}+\Gamma_{J K}^{I} X^{K} \\
& \nabla_{J} X_{I}=\frac{\partial X_{I}}{\partial \Phi^{J}}-\Gamma_{J I}^{K} X_{K}
\end{aligned}
$$

with straightforward generalization to higher order tensors. Anything constructed out of field-space tensors and the field-space covariant derivative will be invariant under (8.2) provided all indices are properly contracted.

\section{THE GRAND CONFIGURATION SPACE}

We now wish to extend the geometric construction of the grand field space in order to take into account the spacetime dependence of the fields. This means that each coordinate now comes with a spacetime argument,

$$
\Phi^{i} \equiv \Phi^{I}\left(x_{I}\right)
$$

As in Sec. II, the lowercase Latin index $i=\left\{I, x_{I}\right\}$ is a continuous index and runs over all points in spacetime as well as all the fields in our theory.

In order to maintain both manifest diffeomorphism and frame invariance, we will make use of the invariant spacetime metric (7.2) and define the spacetime line element as in (7.4). We will also use the corresponding invariant volume element when performing spacetime integrals and from now on, integrations of repeated configuration-space indices will be performed as

$$
X_{i} Y^{i} \equiv \sum_{I} \int d^{D} x_{I} \sqrt{-\bar{g}} X_{I}\left(x_{I}\right) Y^{I}\left(x_{I}\right) .
$$

This choice of spacetime metric directly affects the definition of both the functional derivative and the functional determinant, and we will be explicit in defining them such that their dependence on the metric is made clear.

With the help of the spacetime metric $\bar{g}_{\mu \nu}$, we can define functional differentiation as follows: $\frac{\bar{\delta} F[\Phi(x)]}{\bar{\delta} \Phi(y)} \equiv \lim _{\epsilon \rightarrow 0} \frac{F\left[\Phi(x)+\epsilon \bar{\delta}^{(D)}(x-y)\right]-F[\Phi(x)]}{\epsilon}$,

where we have defined

$$
\bar{\delta}^{(D)}(x) \equiv \ell^{D} \delta^{(D)}(x)
$$

such that

$$
\int d^{D} x \sqrt{-\bar{g}} \bar{\delta}^{(D)}(x)=1
$$

With the definition (9.4), $\bar{\delta}^{(D)}(x)$ is both diffeomorphism and frame invariant. As a result, functional derivatives defined as in (9.3) will inherit their transformation properties from the functional $F$ and field $\Phi$.

Notice that in general $\bar{g}_{\mu \nu}$ depends on all of the grand field-space coordinates $\Phi^{I}$ and therefore so does $\bar{\delta}^{(D)}(x)$. This means that derivatives of the form

$$
\frac{\bar{\delta}}{\bar{\delta} \Phi^{i}} \bar{\delta}^{(D)}(x) \neq 0
$$

will be nonzero. This is not just a consequence of the definition (7.2). Even with the standard noninvariant definitions (i.e., with $g_{\mu \nu}$ identified as the metric), the diffeomorphism-invariant Dirac delta function cannot be treated as a constant once the metric is dynamical.

The condition (9.6) causes the functional derivative (9.3) not to commute. This is not a problem. As the calculation in Sec. V shows, Feynman rules must be calculated in a symmetric way and therefore there is no ambiguity stemming from the order of derivatives. Furthermore, $\bar{\delta}^{(D)}(x)$ has no dependence on $\partial_{\mu} \Phi^{i}$ and so the definition of the configuration-space metric (2.7) can be generalized straightforwardly as shown below.

The choice of metric $\bar{g}_{\mu \nu}$ also affects how we take the functional determinant, since for an infinite-dimensional matrix, the determinant involves an integral over the continuous degrees of freedom. We must therefore explicitly choose which volume measure we will use to count them. Using the invariant volume element derived from (7.4), the functional determinant is given by

$$
\overline{\operatorname{det}}\left(M_{x y}\right) \equiv \exp \left[i \int d^{D} x \sqrt{-\bar{g}} \ln (M)_{x x}\right] .
$$

We have written both the functional derivative and the functional determinant with an overbar to emphasize that these are defined with respect to the metric $\bar{g}_{\mu \nu}$. Using any other metric (e.g., $g_{\mu \nu}$ ) would lead to a nonequivalent definition and, in general, would not maintain diffeomorphism and frame invariance.

These definitions allow us to define the metric of the grand configuration space as follows: 


$$
\begin{aligned}
\mathcal{G}_{i j} & \equiv \frac{\bar{g}_{\mu \nu}}{D} \overline{\bar{\delta}\left(\partial_{\mu} \Phi^{i}\right) \bar{\delta}\left(\partial_{\nu} \Phi^{j}\right)} \\
& =G_{I J}\left(x_{I}\right) \bar{\delta}^{(D)}\left(x_{I}-x_{J}\right) .
\end{aligned}
$$

The uniqueness of the configuration-space metric was questioned by DeWitt (see discussion in Sec. 14 of [40]). Indeed, without the introduction of the model function $\ell$, there would be an ambiguity as to which spacetime metric should be used in the definition (9.8) [17]. In our prescription, however, $\ell$ is a fundamental part of the theory, no less important than $f, k_{A B}$, or $V$. Therefore, for a given theory, $\ell$ must have a fixed functional form and hence the definition (9.8) is unique. Nonetheless, as we discuss below, one needs to take care in eliminating any further dependence of the effective action on gauge-fixing conditions.

With the help of the grand configuration-space metric, we may write down the line element of the grand configuration space as

$$
\begin{aligned}
\mathcal{D} \Sigma^{2}[\boldsymbol{\Phi}] & \equiv \mathcal{G}_{i j} \mathcal{D} \Phi^{i} \mathcal{D} \Phi^{j} \\
& =\int d^{D} x \sqrt{-\bar{g}} G_{I J}(x) \mathcal{D} \Phi^{I}(x) \mathcal{D} \Phi^{J}(x) .
\end{aligned}
$$

We can also construct the configuration-space connection, given by the Christoffel symbols

$$
\Gamma_{j k}^{i} \equiv \frac{1}{2} \mathcal{G}^{i l}\left[\frac{\bar{\delta} \mathcal{G}_{j l}}{\bar{\delta} \Phi^{k}}+\frac{\bar{\delta} \mathcal{G}_{l k}}{\bar{\delta} \Phi^{j}}-\frac{\bar{\delta} \mathcal{G}_{j k}}{\bar{\delta} \Phi^{l}}\right]
$$

and hence a covariant functional derivative

$$
\begin{aligned}
& \bar{\nabla}_{j} X^{i}=\frac{\bar{\delta} X^{i}}{\bar{\delta} \Phi^{j}}+\Gamma_{j k}^{i} X^{k}, \\
& \bar{\nabla}_{j} X_{i}=\frac{\bar{\delta} X_{i}}{\bar{\delta} \Phi^{j}}-\Gamma_{j i}^{k} X_{k},
\end{aligned}
$$

with straightforward generalization to higher order tensors.

The invariant configuration-space line element allows us to construct an invariant path-integral volume element

$$
[\overline{\mathcal{D}} \boldsymbol{\Phi}] \sqrt{\overline{\operatorname{det}}\left(\mathcal{G}_{i j}\right)} .
$$

Note that the functional integral element $[\overline{\mathcal{D}} \boldsymbol{\Phi}]=$ $\prod_{x, I} d \Phi^{I}(x)$ is the product of integral elements at every point in spacetime. How these points are counted depends crucially on the metric of spacetime and therefore $[\overline{\mathcal{D}} \boldsymbol{\Phi}]$ will depend on the model function $\ell$. We have highlighted this by denoting it with an overbar to emphasize the choice of $\bar{g}_{\mu \nu}$ as the metric of spacetime.

We can see the dependence explicitly using the identity $\prod_{i} A_{i}=\exp \left(\sum_{i} \ln \left(A_{i}\right)\right)$, which holds for discrete products and can be extrapolated to continuous products. We therefore have
$[\overline{\mathcal{D}} \boldsymbol{\Phi}]=\exp \left[\sum_{I} \int d^{D} x \sqrt{-\bar{g}(x)} \ln \left(\mathcal{D} \Phi^{I}(x)\right)\right]$

Finally, we must ensure that gauge fixing is done in a reparametrization-invariant manner. We therefore modify the gauge-fixing term (6.3) to be

$$
S_{\mathrm{GF}}[\boldsymbol{\Phi}]=-\frac{\gamma}{2} \int d^{D} x \sqrt{-\bar{g}} \chi^{\mu}(\boldsymbol{\Phi}) \bar{g}_{\mu \nu} \chi^{\nu}(\boldsymbol{\Phi}) .
$$

Defined in this way, the gauge-fixing condition $\chi^{\mu}(\boldsymbol{\Phi})$ is a grand configuration-space scalar.

We note that the gauge-fixing condition is, in general, a function of not only the tensor field $g_{\mu \nu}$, but also the scalar fields $\phi$. Even if a gauge condition $\chi^{\mu}$ that depends only on $g_{\mu \nu}$ is chosen in some frame (e.g., the De Donder gauge used earlier), it will pick up a dependence on $\phi$ after a frame transformation.

At this stage, one may worry whether the gauge-fixing term in (9.14) will threaten the uniqueness of (9.8). However, previous works in the literature $[29,44,46,48,68,69]$ have shown that it is possible to define a projected configurationspace metric leading to an effective action which is independent of the gauge-fixing condition. Therefore, one can employ the techniques developed in these earlier works, as well as the model function $\ell(\boldsymbol{\phi})$ to define such a projected configuration-space metric in a well-defined and unique manner.

Gauge fixing also requires us to include the FaddeevPoppov determinant [78] in our path-integral measure. This can be defined in a frame-invariant way as

$$
V_{\mathrm{FP}}=\overline{\operatorname{det}}\left(\frac{\bar{\delta} \chi^{\mu}(\boldsymbol{x})}{\bar{\delta} \xi^{\nu}(\boldsymbol{y})}\right)
$$

where $\xi^{\mu}$ are the gauge parameters. With this term included, we see that the path-integral measure is

$$
\mathcal{M}[\boldsymbol{\Phi}]=V_{\mathrm{FP}} \sqrt{\overline{\operatorname{det}\left(\mathcal{G}_{i j}\right)}} .
$$

We can use the above constructions to define a diffeomorphism and frame-invariant effective action,

$$
\begin{aligned}
& \exp \left(\frac{i}{\hbar} \Gamma[\boldsymbol{\varphi}]\right) \\
& \quad=\int[\overline{\mathcal{D}} \boldsymbol{\Phi}] \mathcal{M}[\boldsymbol{\Phi}] \exp \left[\frac{i}{\hbar}\left(S[\boldsymbol{\Phi}]+\frac{\bar{\delta} \Gamma[\boldsymbol{\varphi}]}{\bar{\delta} \varphi^{i}} \Sigma^{i}[\boldsymbol{\varphi}, \boldsymbol{\Phi}]\right)\right],
\end{aligned}
$$

where $\boldsymbol{\varphi}=\left(g^{\mu \nu}, \boldsymbol{\phi}\right)$ collectively denotes the grand fields of (8.1) and $S[\boldsymbol{\Phi}]$ includes both the classical action (1.1) and the gauge-fixing term (9.14). The effective action defined in this way satisfies the important property 


$$
\begin{aligned}
& \Gamma\left[\boldsymbol{\varphi} ; \ell(\boldsymbol{\phi}), f(\boldsymbol{\phi}), k_{A B}(\boldsymbol{\phi}), V(\boldsymbol{\phi})\right] \\
& \quad=\Gamma\left[\tilde{\boldsymbol{\varphi}}(\boldsymbol{\varphi}) ; \tilde{\ell}(\boldsymbol{\phi}), \tilde{f}(\boldsymbol{\phi}), \tilde{k}_{A B}(\boldsymbol{\phi}), \tilde{V}(\boldsymbol{\phi})\right] .
\end{aligned}
$$

In (9.18), the transformations of $\tilde{\boldsymbol{\varphi}}, \tilde{\ell}$, and $\left(\tilde{f}, \tilde{k}_{A B}, \tilde{V}\right)$ are given by (8.2), (7.3), and (1.6), respectively. Thus, by construction, $\Gamma$ is manifestly frame invariant.

Given that the configuration-space metric $G_{i j}$ depends on the definition of $\bar{g}_{\mu \nu}$, there will be nontrivial effects of the model function $\ell(\boldsymbol{\phi})$ at the quantum level arising from the measure (9.12).

These effects are equivalent to those calculated in [17]. However, here we provide an alternative interpretation. Instead of arising from a mismatch between the frame that we choose to work in and some preferred frame in which the theory is quantized, the "frame discriminant" is a simply the one-loop effects of the model function $\ell(\boldsymbol{\phi})$. In our approach, the choice of measure does not introduce an ambiguity in the definition of the effective action. Instead, it is part of the theory itself. Therefore, theories with different measures will have different but unique effective actions.

\section{SUMMARY OF THE FRAME COVARIANT FORMALISM}

In this section, we summarize our frame covariant formalism for scalar-tensor theories. To fully specify a scalar-tensor theory, we require the following four model functions:

(1) The effective Planck length $\ell$

(2) The effective Planck mass $f$

(3) The scalar field-space metric $k_{A B}$

(4) The scalar potential $V$

In detail, with these model functions, the classical action is given by

$$
\begin{aligned}
S[\boldsymbol{\Phi}]= & \int d^{D} x \sqrt{-g}\left[-\frac{f R}{2}+\frac{k_{A B}}{2} \partial_{\mu} \phi^{A} \partial^{\mu} \phi^{B}-V\right] \\
& +S_{\mathrm{GF}}[\mathbf{\Phi}],
\end{aligned}
$$

where $\boldsymbol{\Phi}$ are the grand fields given by (8.1), spacetime indices are contracted with $g_{\mu \nu}$, and $S_{\mathrm{GF}}$ is given by (9.14). We can then extract the metric of the grand configuration space, $\mathcal{G}_{i j}$, from the classical action using

$$
\mathcal{G}_{i j}[\mathbf{\Phi}]=\frac{\bar{g}_{\mu \nu}}{D} \frac{\bar{\delta}^{2} S[\boldsymbol{\Phi}]}{\bar{\delta}\left(\partial_{\mu} \Phi_{I}\left(x_{I}\right)\right) \bar{\delta}\left(\partial_{\nu} \Phi_{J}\left(x_{J}\right)\right)},
$$

where $\bar{g}_{\mu \nu}=g_{\mu \nu} / \ell^{2}$ is the metric of spacetime as given in (7.2).

We can calculate the quantum effects of this theory in two equivalent ways. One way is to use the VDW action $\Gamma[\boldsymbol{\varphi}]$. This can be calculated from the implicit equation (9.17).
An alternative way in which quantum corrections can be calculated is through the use of covariant Feynman diagrams as described in Sec. V. In this approach, Feynman rules are calculated in a covariant manner with the propagators given by

$$
i \bullet j=\left(\left.\nabla_{i} \nabla_{j} S\right|_{\Phi_{0}}\right)^{-1}
$$

and the vertices given by

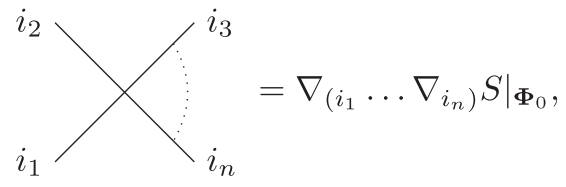

where $\boldsymbol{\Phi}_{0}$ is the base point of the perturbation, usually taken to be the classical vacuum. Feynman diagrams can then be calculated in the usual way.

Both of the above approaches agree with the standard calculation for on-shell observables, but they additionally preserve reparametrization invariance off shell.

\section{CONCLUSIONS}

We have developed a covariant formalism for scalartensor theories of quantum gravity. By extending the Vilkovisky-DeWitt effective action and the geometric structure of the configuration space, we have constructed a quantum field theory that is manifestly frame and spacetime-diffeomorphism invariant.

This is in contrast to previous approaches, which required us to identify a preferred frame in which the expression for the ordinary effective action (3.4) holds. The noncovariance of (3.4) leads to an inequivalence in the standard approach between theories with different choices of preferred frame. This is the root of the cosmological frame problem.

Our formalism resolves this issue by identifying a new model function $\ell(\boldsymbol{\phi})$ that relates the spacetime metric $\bar{g}_{\mu \nu}$ and the gravitational tensor field $g_{\mu \nu}$. Choosing the form of $\ell(\boldsymbol{\phi})$ in our formalism is equivalent to choosing a preferred frame in the conventional approach but does not unduly privilege a particular frame.

In addition, we have seen how the choice of spacetime metric affects the contraction of DeWitt indices, the definition of functional determinants as well as the normalization of the Dirac delta function, and the definition of functional derivatives. In many cases, the $\ell$ dependence in these definitions will cancel out and the results of calculations using our conventions will reduce to those obtained using the standard definitions involving $g_{\mu \nu}$. Indeed, we see that the only dependence of $\ell$ in (9.17) that does not cancel is in the definition of the configuration-space metric (9.8).

However, the conventions we have laid out in this paper are essential if we want to keep both diffeomorphism invariance and frame invariance manifest. Without a 
frame-invariant definition of the spacetime metric, it would be impossible to define configuration-space tensors that transform correctly. For example, the standard functional derivative $\delta F / \delta \Phi^{i}$ does not transform as a configurationspace vector, even if $F$ is a configuration-space scalar. In addition, in order to obtain frame covariant correlation functions from the effective action, we must use the covariant functional derivatives as defined in (9.11).

The freedom of choosing a preferred frame still exists in our formalism. However, it is now explicitly part of the content of the theory, captured by the model function $\ell$, as opposed to being expressed by singling out a particular parametrization. After all, the relation between the tensor field $g_{\mu \nu}$ and the metric of spacetime $\bar{g}_{\mu \nu}$ is a physical one and not just a convention. Two theories with the same classical action, but a different relation between $g_{\mu \nu}$ and $\bar{g}_{\mu \nu}$ cannot be related by a frame transformation (8.2) and will, in general, give rise to different quantum predictions.

Our formalism therefore draws a clear dividing line between the content of a theory and its representation. Once we have picked a particular form for the model functions $f$, $k_{A B}, V$, and $\ell$, we have uniquely specified our QFT and therefore all of its physical predictions. However, we may still change the representation of the theory by performing a frame transformation (8.2). The model functions will be different after this change of frame, but the QFT as defined by $\Gamma[\varphi]$ will still have the same functional form as shown in (9.18) and will make the same predictions.

We note that when $\ell$ is treated as a model function, the definition (9.8) determines the configuration-space metric in a way that does not depend on the parametrization and does not rely on any preferred frame. Although we have not discussed it here in detail, previous works $[29,44,46,48,68,69]$ have shown that any dependence on the gauge-fixing condition can also be removed. Hence, the configuration-space metric for a scalar-tensor theory can be uniquely defined. Once the configuration space has been defined, the definition (9.17) fully determines the VDW effective action. Consequently, the VDW effective action is uniquely determined from the four model functions $f, k_{A B}$, $V$, and $\ell$.

Since the covariant quantum effective action (9.17) is frame invariant and the ordinary effective action (3.4) is not, it is clear that they can only agree in at most one frame. This frame is one in which $\ell=1$ and additionally all the scalar fields are canonically normalized. However, such a canonical frame does not exist for theories with intrinsic field-space curvature. Thus, for such theories, the usual approach is not suitable in any frame and we must adopt the formalism developed in this paper in order to maintain reparametrization invariance.

This observation may be important for the development of a UV-complete quantum theory of gravity. The EinsteinHilbert action for gravity (6.1) is nonrenormalizable, which has long prevented such a theory being constructed. As we have shown in Sec. VI, general relativity features a curved field space. This curvature alters the calculation of quantum corrections and must therefore be taken into account in order to UV complete the theory.

By identifying the model function $\ell$, we have identified the source of the cosmological frame problem. It is not possible to write down a unique effective action without specifying the form of $\ell$, and any formalism that does not include this model function will have an inherent ambiguous choice of frame. Any frame transformation that does not take into account the transformation of $\ell$ will lead to a different theory with different quantum predictions. This implies that the classical action is not sufficient to fully define a QFT.

In this paper, we have taken reparametrization invariance as a fundamental guiding light. We argue that a theory should not depend on the way it is parametrized, and therefore Lagrangians related by a frame transformation are different expressions of the same underlying theory. Based on this idea, we have developed a formalism in which the invariance of physical predictions under such field reparametrizations is made manifest. Our formalism can be used to derive a quantum effective action that is manifestly invariant under frame transformations that include $\ell$.

\section{ACKNOWLEDGMENTS}

The authors would like to thank Jack Holguin and Chris Shepherd for useful comments and discussion. We would also like to thank Daniel Martin for his help in writing the Mathematica code used to calculate covariant Feynman rules. The work of K. F. was supported by the University of Manchester through the President's Doctoral Scholar Award. The work of A. P. and S. K. was supported by the LancasterManchester-Sheffield Consortium for Fundamental Physics under STFC research Grant No. ST/L000520/1.

\section{APPENDIX A: THEORY WITH A COMPLEX SCALAR FIELD}

As an example to highlight the parametrization dependence of the standard formulation of QFTs, we consider the example of a single complex scalar field $\phi$ with action

$$
S=\int d^{4} x\left[\partial_{\mu} \phi \partial^{\mu} \phi-m^{2}|\phi|^{2}-\lambda|\phi|^{4}\right] .
$$

We choose our parameters with $m^{2}<0$ so that the vacuum is

$$
\langle\phi\rangle=\frac{1}{\sqrt{2}} \rho_{0} \equiv \sqrt{\frac{-m^{2}}{2 \lambda}} .
$$

This theory has $U(1)$ symmetry $\phi \rightarrow e^{i \theta} \phi$, which is spontaneously broken by the vacuum (A2). Therefore, the perturbations will have two modes, a massive Higgs mode and a massless Goldstone mode.

For simplicity, we will assume a flat, static, background spacetime with Minkowski metric $\eta_{\mu \nu}=\operatorname{diag}(1,-1,-1,-1)$. 


\section{Standard approach: Linear parametrization}

The complex field $\phi$ contains 2 real degrees of freedom, which we can parametrize in terms of its real and imaginary parts as

$$
\phi=\frac{\phi_{1}+i \phi_{2}}{\sqrt{2}} .
$$

In this parametrization, the action (A1) is

$$
\begin{aligned}
S= & \int d^{4} x\left[\frac{1}{2} \partial_{\mu} \phi_{1} \partial^{\mu} \phi_{1}+\frac{1}{2} \partial_{\mu} \phi_{2} \partial^{\mu} \phi_{2}\right. \\
& \left.-\frac{1}{2} m^{2}\left(\phi_{1}^{2}+\phi_{2}^{2}\right)-\frac{\lambda}{4}\left(\phi_{1}^{2}+\phi_{2}^{2}\right)^{2}\right]
\end{aligned}
$$

and the vacuum (A2) is

$$
\left\langle\phi_{1}\right\rangle=\rho_{0}, \quad\left\langle\phi_{2}\right\rangle=0
$$

As stated before, the perturbations consist of a massive Higgs mode, corresponding to perturbations of $\phi_{1}$, and a massless Goldstone mode corresponding to perturbations of $\phi_{2}$.

\section{a. Effective potential}

Let us start by calculating the one-loop correction to the effective action via (3.5). The inverse propagator for this theory is

$$
\frac{\delta^{2} S}{\delta \phi^{A}(x) \delta \phi^{B}(y)}=\left(\begin{array}{cc}
-\partial^{2}-m^{2}-3 \lambda \phi_{1}^{2}-\lambda \phi_{2}^{2} & -2 \lambda \phi_{1} \phi_{2} \\
-2 \lambda \phi_{1} \phi_{2} & -\partial^{2}-m^{2}-3 \lambda \phi_{2}^{2}-\lambda \phi_{1}^{2}
\end{array}\right) \delta^{(4)}(x-y) .
$$

Without loss of generality, we can use the $U(1)$ symmetry to set $\phi_{2}=0$. Thus, the one-loop effective action evaluated for a static configuration (the effective potential) in the $\overline{\mathrm{MS}}$ renormalization scheme is

$$
\begin{aligned}
V_{\mathrm{eff}}(\varphi) & \equiv-\frac{1}{V_{4}} \Gamma\left[\phi_{1}=\varphi, \phi_{2}=0\right] \\
& =V(\varphi)-\frac{i}{2} \ln \operatorname{det} G_{A B}+\frac{i}{2} \ln \operatorname{det}\left[\partial^{2}+m^{2}+3 \lambda \varphi^{2}\right]+\frac{i}{2} \ln \operatorname{det}\left[\partial^{2}+m^{2}+\lambda \varphi^{2}\right] \\
& =\frac{1}{2} m^{2} \varphi^{2}+\frac{1}{4} \lambda \varphi^{4}+\frac{1}{64 \pi^{2}}\left\{\left(m^{2}+3 \lambda \varphi^{2}\right)^{2}\left[\ln \left(\frac{m^{2}+3 \lambda \varphi^{2}}{\mu^{2}}\right)-\frac{3}{2}\right]+\left(m^{2}+\lambda \varphi^{2}\right)^{2}\left[\ln \left(\frac{m^{2}+\lambda \varphi^{2}}{\mu^{2}}\right)-\frac{3}{2}\right]\right\},
\end{aligned}
$$

where $V_{4}$ is the total four-volume of spacetime. Notice that, for a static configuration, $\ln \operatorname{det} G_{A B}=0$ in dimensional regularization.

\section{b. Feynman rules and renormalization}

The standard calculation (5.11) leads to the following Feynman rules:

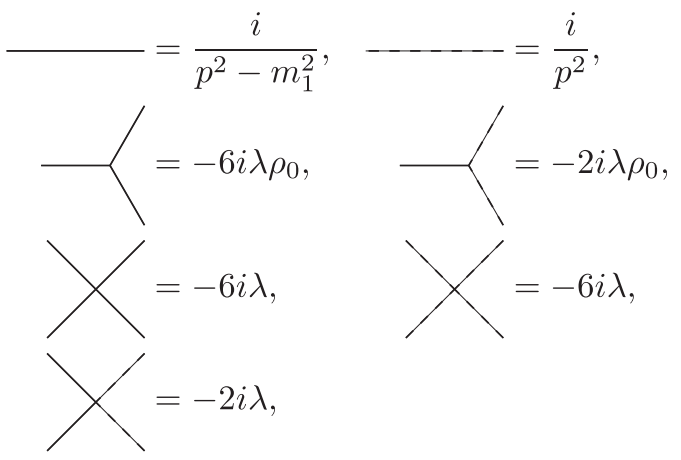

where

$$
m_{1}^{2} \equiv m^{2}+3 \lambda \rho_{0}^{2}=-2 m^{2}
$$

is the mass of the Higgs mode. Here we represent the Higgs mode $\phi_{1}$ by a solid line and the Goldstone mode $\phi_{2}$ by a dashed line.

Let us use these Feynman rules to calculate the renormalization of the Higgs mass. At one-loop order, we have

$$
\begin{aligned}
= & i\left(p^{2}-m_{1}^{2}\right)+\frac{3 i \lambda}{(4 \pi)^{2}} A\left(m_{1}^{2}\right)+\frac{i \lambda}{(4 \pi)^{2}} A(0) \\
& +18 i \frac{\lambda^{2} \rho_{0}^{2}}{(4 \pi)^{2}} B_{0}\left(p^{2}, m_{1}, m_{1}\right)+2 i \frac{\lambda^{2} \rho_{0}^{2}}{(4 \pi)^{2}} B_{0}\left(p^{2}, 0,0\right) \\
& -18 i \frac{\lambda^{2} \rho_{0}^{2}}{(4 \pi)^{2} m_{1}^{2}} A\left(m_{1}^{2}\right)-6 i \frac{\lambda^{2} \rho_{0}^{2}}{(4 \pi)^{2} m_{1}^{2}} A(0) .
\end{aligned}
$$


Here we have defined the following two integrals:

$$
\begin{gathered}
A\left(m^{2}\right) \equiv \int \frac{d^{4} k}{i \pi^{2}} \frac{1}{k^{2}-m^{2}}, \\
B_{0}\left(p^{2}, m_{1}, m_{2}\right) \equiv \int \frac{d^{4} k}{i \pi^{2}} \frac{1}{k^{2}-m_{1}^{2}} \frac{1}{(p+k)^{2}-m_{2}^{2}},
\end{gathered}
$$

which we can perform using dimensional regularization scheme to give

$$
\begin{array}{r}
A\left(m^{2}\right)=m^{2}\left[C_{\mathrm{UV}}+1-\ln \left(\frac{m^{2}}{\mu^{2}}\right)\right], \quad(\mathrm{A} 12) \\
B_{0}\left(p^{2}, m_{1}, m_{2}\right) \\
=C_{\mathrm{UV}}-\int_{0}^{1} d x \ln \left(\frac{m_{1}^{2}(1-x)+m_{2}^{2} x-x(1-x) p^{2}}{\mu^{2}}\right),
\end{array}
$$

where $\mu$ is the renormalization scale and

$$
C_{\mathrm{UV}}=\frac{2}{4-D}-\gamma_{\mathrm{E}}+\ln (4 \pi)
$$

is the UV divergence that is cancelled by counterterms in the $\overline{\mathrm{MS}}$ renormalization scheme. Here $D=4-2 \epsilon$, and $\gamma_{\mathrm{E}}=0.577 \ldots$ is the Euler-Mascheroni constant. We therefore have

$$
\begin{aligned}
\Gamma_{\phi_{1} \phi_{1}}(p)= & \left(p^{2}-m_{1}^{2}\right)+\frac{\lambda m_{1}^{2}}{4 \pi^{2}} \ln \left(\frac{p^{2}}{\mu^{2}}\right) \\
& -\frac{\lambda m_{1}^{2}}{(4 \pi)^{2}}\left[-4 C_{\mathrm{UV}}+4\right. \\
& \left.+9 \int_{0}^{1} d x \ln \left(\frac{x(x-1) p^{2}+m_{1}^{2}}{\mu^{2}}\right)\right] .
\end{aligned}
$$

Note that $A(0)=0$ and thus the third and final diagrams in (A9) give no contribution.

From (A15), we see that there is no wave function renormalization, as expected, and the beta function of the Higgs mass is

$$
\beta_{m_{1}^{2}}=-\mu \frac{\partial \hat{\Gamma}_{2}}{\partial \mu}=\frac{\lambda m_{1}^{2}}{2 \pi^{2}}
$$

We can also calculate the Goldstone self-energy. At one loop, we have

$$
\begin{aligned}
i \Gamma_{\phi_{2} \phi_{2}}(p) \\
=i p^{2}+\frac{i \lambda}{(4 \pi)^{2}} A\left(m_{1}^{2}\right)+\frac{3 i}{(4 \pi)^{2}} \lambda A(0) \\
+4 i \frac{\lambda^{2} \rho_{0}^{2}}{(4 \pi)^{2}} B_{0}\left(p^{2}, m_{1}, 0\right)-6 i \frac{\lambda^{2} \rho_{0}^{2}}{(4 \pi)^{2} m_{1}^{2}} A\left(m_{1}^{2}\right) \\
-2 i \frac{\lambda^{2} \rho_{0}^{2}}{(4 \pi)^{2} m_{1}^{2}} A(0) \\
=i p^{2}-\frac{2 i \lambda m_{1}^{2}}{16 \pi^{2}} \int_{0}^{1} d x \ln \left(1-\frac{x p^{2}}{m_{1}^{2}}\right) .
\end{aligned}
$$

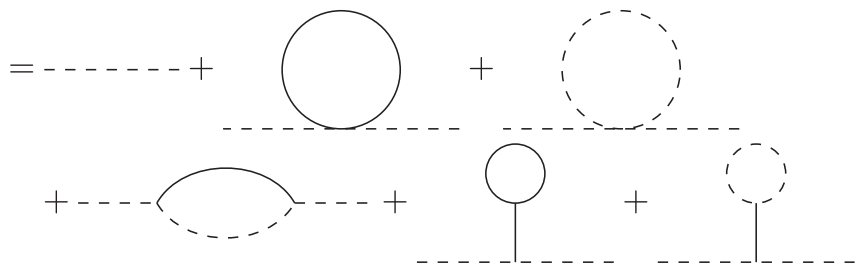

Since (A17) has no dependence on $\mu$, the Goldstone mass is not renormalized and remains zero in accordance with Goldstone's theorem.

Finally, let us compute the coupling renormalization using the Callan-Symanzic equation $[79,80]$

$$
\left[\mu \frac{\partial}{\partial \mu}+\beta_{\lambda} \frac{\partial}{\partial \lambda}+\beta_{m^{2}} \frac{\partial}{\partial m^{2}}\right] \tilde{V}_{\text {eff }}=0,
$$

where

$$
\tilde{V}_{\text {eff }}(\varphi)=V_{\text {eff }}(\varphi)-V_{\text {eff }}(0)
$$

is the modified effective potential. From the expression for $V_{\text {eff }}$ in (A6), we have, at leading order,

$$
\begin{aligned}
\frac{1}{4} \beta_{\lambda} \varphi^{4}-\frac{1}{4} \beta_{m_{1}^{2}} \varphi^{2} & \\
-\frac{\left(m^{2}+3 \lambda \varphi^{2}\right)^{2}+\left(m^{2}+\lambda \varphi^{2}\right)^{2}-2 m^{4}}{32 \pi^{2}} & =0,
\end{aligned}
$$

where we have used the identity $\beta_{m_{1}^{2}}=-2 \beta_{m^{2}}$, which derives from (A8).

Rearranging and using the expression for $\beta_{m_{1}^{2}}$ from (A16), we see that the beta function for the coupling renormalization, evaluated at the vacuum $\varphi=\rho_{0}$, is

$$
\beta_{\lambda}=\frac{5}{4 \pi^{2}} \lambda^{2}
$$

\section{Standard approach: Nonlinear parametrization}

Alternatively, we could have used a nonlinear parametrization of the complex field 


$$
\phi=\frac{1}{\sqrt{2}} \rho e^{i \frac{\sigma}{\rho_{0}}}
$$

In this parametrization, the action (A1) is

$$
S=\int d^{4} x\left[\frac{1}{2} \partial_{\mu} \rho \partial^{\mu} \rho+\frac{1}{2}\left(\frac{\rho}{\rho_{0}}\right)^{2} \partial_{\mu} \sigma \partial^{\mu} \sigma-\frac{1}{2} m^{2} \rho^{2}-\frac{\lambda}{4} \rho^{4}\right]
$$

and the vacuum (A2) is

$$
\langle\rho\rangle=\rho_{0}, \quad\langle\sigma\rangle=0 .
$$

In this parametrization, the Higgs mode is in the direction of $\rho$ and the Goldstone mode is in the direction of $\sigma$.

\section{a. Effective potential}

Let us calculate the one-loop effective action in this parametrization using (3.5). The inverse propagator in this parametrization is

$$
\frac{\delta^{2} S}{\delta \phi^{a}(x) \delta \phi^{b}(y)}=\left(\begin{array}{cc}
-\partial^{2}+\partial_{\mu} \sigma \partial^{\mu} \sigma-m^{2}-3 \lambda \rho^{2} & -2 \partial_{\mu} \rho \partial^{\mu} \sigma-2 \rho \partial^{2} \sigma-2 \rho \partial_{\mu} \sigma \partial^{\mu} \\
-2 \partial_{\mu} \rho \partial^{\mu} \sigma-2 \rho \partial^{2} \sigma-2 \rho \partial_{\mu} \sigma \partial^{\mu} & -2 \rho \partial_{\mu} \rho \partial^{\mu}-\rho^{2} \partial^{2}
\end{array}\right) \delta^{(4)}(x-y) .
$$

As before we can, without loss of generality, use the $U(1)$ symmetry to set $\sigma=0$. Again, we will consider a static configuration in order to calculate the effective action. In the $\overline{\mathrm{MS}}$ scheme, this is given by

$$
\begin{aligned}
V_{\mathrm{eff}}(\varphi) & \equiv-\frac{1}{V_{4}} \Gamma[\rho=\varphi, \sigma=0] \\
& =\frac{1}{2} m^{2} \varphi^{2}+\frac{\lambda}{4} \varphi^{4}-\ln \operatorname{det}\left[\partial^{2}+m^{2}+3 \lambda \varphi^{2}\right]-\ln \operatorname{det}\left[\varphi^{2} \partial^{2}\right] \\
& =\frac{1}{2} m^{2} \varphi^{2}+\frac{\lambda}{4} \varphi^{4}+\frac{1}{64 \pi^{2}}\left\{\left(m^{2}+3 \lambda \varphi^{2}\right)^{2}\left[\ln \left(\frac{m^{2}+3 \lambda \varphi^{2}}{\mu^{2}}\right)-\frac{3}{2}\right]\right\} .
\end{aligned}
$$

Notice that (A6) and (A26) differ off shell, highlighting the parametrization dependence of the standard effective action. However, on shell when $\varphi=\rho_{0}=\sqrt{-m^{2} / \lambda}$, the two expressions agree.

Note that if we had instead taken $m^{2}>0$, the vacuum would lie at $\varphi=0$. Surprisingly, in this case, (A6) and (A26) do not agree even on shell. This is due to a peculiarity with the particular coordinate chart (A22). The point $\phi=0$ is multiply covered by this chart and therefore represents a coordinate singularity-a point where the chart cannot be trusted.

To rectify this problem, we can define an offset parametrization $\phi=\frac{1}{\sqrt{2}}\left(\tilde{\rho} e^{i \tilde{\sigma}}-\delta\right)$ so that the vacuum $\phi=0$ is no longer at the singular point. In the offset parametrization, the effective potential is

$$
\begin{aligned}
V_{\mathrm{eff}}(\tilde{\rho}=\tilde{\varphi}, \tilde{\sigma}=0) \\
=\frac{1}{2} m^{2}(\tilde{\varphi}-\delta)^{2}+\frac{\lambda}{4}(\tilde{\varphi}-\delta)^{4} \\
\quad+\frac{\left(m^{2}+3 \lambda(\tilde{\varphi}-\delta)^{2}\right)^{2}}{64 \pi^{2}}\left[\ln \left(\frac{m^{2}+3 \lambda(\tilde{\varphi}-\delta)^{2}}{\mu^{2}}\right)-\frac{3}{2}\right] \\
\quad+\frac{\delta^{2}}{\tilde{\varphi}^{2}} \frac{\left(m^{2}+\lambda(\tilde{\varphi}-\delta)^{2}\right)^{2}}{64 \pi^{2}}\left[\ln \left(\frac{\delta}{\tilde{\varphi}} \frac{m^{2}+\lambda(\tilde{\varphi}-\delta)^{2}}{\mu^{2}}\right)-\frac{3}{2}\right],
\end{aligned}
$$

which we can see does agree with (A6) at $\tilde{\varphi}=\delta$. Thus, in order to calculate the effective action (A26) at $\varphi=0$, we should take the limit $\tilde{\varphi} \rightarrow \delta \rightarrow 0$ in (A27), which gives us

$$
V_{\text {eff }}(0)=\frac{2 m^{2}}{64 \pi^{2}}\left[\ln \left(\frac{m^{2}}{\mu^{2}}\right)-\frac{3}{2}\right]
$$

in agreement with (A6). This expression will be needed for the Callan-Symanzic equation.

\section{b. Feynman rules and renormalization}

The standard Feynman rules from (5.11) in this parametrization are

$$
\begin{aligned}
& =\frac{i}{p^{2}-m_{1}^{2}}, \quad \ldots=\frac{i}{p^{2}}, \\
& =-6 i \lambda \rho_{0}, \quad \longrightarrow=-6 i \lambda, \quad k_{1}=-\frac{2 i}{\rho_{0}} k_{1} \cdot k_{2}, \\
& k_{k_{2}}
\end{aligned}
$$

As before, the solid line represents the Higgs mode and the dashed line represents the Goldstone mode. As expected, these Feynman rules are different from (A7), showing explicitly the parametrization noninvariance of this approach.

If we calculate the Higgs mass renormalization with this parametrization, we will find 


$$
\begin{aligned}
i \Gamma_{\rho \rho}(p)= & \\
= & i\left(p^{2}-m_{1}^{2}\right)+\frac{3 i \lambda}{(4 \pi)^{2}} A\left(m_{1}^{2}\right)+\frac{2 i}{\rho_{0}} \int \frac{d^{4} k}{(2 \pi)^{4}} \\
& +18 i \frac{\lambda^{2} \rho_{0}^{2}}{(4 \pi)^{2}} B_{0}\left(p^{2}, m_{1}, m_{1}\right)+\frac{i}{2} \frac{p^{4}}{(4 \pi)^{2} \rho_{0}^{2}} B_{0}\left(p^{2}, 0,0\right) \\
& -18 i \frac{\lambda^{2} \rho_{0}^{2}}{(4 \pi)^{2} m_{1}^{2}} A\left(m_{1}^{2}\right)+6 i \frac{\lambda^{2} \rho_{0}^{2}}{m_{1}^{2}} \int \frac{d^{4} k}{(2 \pi)^{4}} \\
= & i\left(p^{2}-m_{1}^{2}\right)+\frac{3 \lambda m_{1}^{2}+\lambda \frac{p^{4}}{m_{1}^{4}}}{(4 \pi)^{2}} \ln \left(\frac{p^{2}}{\mu^{2}}\right) \\
& +\frac{i \lambda m_{1}^{2}}{(4 \pi)^{2}}\left[\left(3+\frac{p^{4}}{m_{1}^{4}}\right) C_{\mathrm{UV}}-6+2 \frac{p^{4}}{m_{1}^{4}}\right. \\
& \left.-9 \int_{0}^{1} d x \ln \left(\frac{x(x-1) p^{2}+m_{1}^{2}}{\mu^{2}}\right)\right]
\end{aligned}
$$

We see that, as expected, this differs from (A15) off shell. In fact, due to the presence of the $p^{4}$ divergence, this theory is naively nonrenormalizable. However, if we only consider on-shell momentum, so that $p^{2}=m_{1}^{2}$ the two expressions, (A15) and (A30), are equal. As a result, the beta function will be given by (A16).

We can also calculate the Goldstone mass renormalization,

$$
\begin{aligned}
i \Gamma_{\sigma \sigma}(p)= & \\
= & i p^{2}-\frac{i p^{2}}{(4 \pi)^{2} \rho_{0}^{2}} A\left(m_{1}^{2}\right) \\
& +6 i \frac{\lambda p^{2}}{(4 \pi)^{2} m_{1}^{2}} A\left(m_{1}^{2}\right)-2 i \frac{p^{2}}{\rho_{0}^{2}} \int \frac{d^{4} k}{(2 \pi)^{4}} \\
& +\left[i \frac{3 p^{2}-m_{1}^{2}}{(4 \pi)^{2} \rho_{0}^{2}} A\left(m_{1}^{2}\right)+i \frac{\left(p^{2}-m_{1}^{2}\right)^{2}}{(4 \pi)^{2} \rho_{0}^{2}} B_{0}\left(p^{2}, m_{1}, 0\right)\right] \\
= & i p^{2}+i \frac{5 p^{2}-m_{1}^{2}}{16 \pi^{2} \rho_{0}^{2}} m_{1}^{2}\left[C_{\mathrm{UV}}+1-\ln \left(\frac{m^{2}}{\mu^{2}}\right)\right] \\
& +i \frac{\left(p^{2}-m_{1}^{2}\right)^{2}}{16 \pi^{2} \rho_{0}^{2}}\left[C_{\mathrm{UV}}\right. \\
& \left.-\int_{0}^{1} d x \ln \left(\frac{(1-x) m_{1}^{2}-x(1-x) p^{2}}{\mu^{2}}\right)\right] .
\end{aligned}
$$

As before, this expression differs from the expression obtained using the linear parametrization (A17) and also contains nonrenormalizable terms. However, on shell, when $p^{2}=0$, we have

$$
\Gamma_{\sigma \sigma}\left(p^{2}=0\right)=0
$$

in agreement with (A17) and the Goldstone mass is not renormalized as expected by Goldstone's theorem.

Finally, we look at the coupling renormalization, which we shall calculate through the Callan-Symanzic equation (A18) as before. Using the expression (A26) for the effective action gives us

$$
-\frac{\left(m^{2}+3 \lambda \varphi^{2}\right)^{2}-2 m^{4}}{32 \pi^{2}}+\frac{1}{4} \beta_{\lambda} \varphi^{4}-\frac{1}{4} \beta_{m_{1}^{2}} \varphi^{2}=0 .
$$

On shell when $\varphi=\rho_{0}$, this becomes identical to (A20), which means that the beta function for the coupling renormalization is

$$
\beta_{\lambda}=\frac{5}{4 \pi^{2}} \lambda^{2}
$$

as before. ${ }^{5}$

Although the two approaches led to several differences in the intermediate, off-shell results, as the above calculation demonstrates, all physical observables are the same regardless of the parametrization.

\section{Covariant approach}

We have shown in the main text how to alleviate the parametrization dependence of quantum calculations by using an explicitly covariant formalism. Let us now repeat the above calculations using this formalism to show how parametrization invariance is maintained.

For the linear parametrization (A3), the field space is trivial, and so there is no difference between the covariant approach and the standard (ordinary) approach. Thus, the VDW effective potential will be (A6), the covariant Feynman rules will give (A7), and the renormalization group calculations will be identical to those in Sec. A 1 .

We will therefore focus on the nonlinear parametrization (A22). In this parametrization, the configuration-space metric (2.7) is

$$
\mathcal{G}_{a b}=\left(\begin{array}{cc}
1 & 0 \\
0 & \left(\rho / \rho_{0}\right)^{2}
\end{array}\right) \delta^{(4)}\left(x_{a}-x_{b}\right),
$$

and the nonzero configuration-space Christoffel symbols can be calculated as

\footnotetext{
${ }^{5}$ Note that we had to use (A27) to calculate $V_{\text {eff }}(\varphi=0)$. Had we used (A26) instead, the two results would not have agreed. As stated earlier, this is because the parametrization (A22) features a coordinate singularity at $\rho=0$ and so cannot be trusted there.
} 


$$
\begin{gathered}
\Gamma_{\sigma(x) \sigma(y)}^{\rho(z)}=-\frac{\rho(z)}{\rho_{0}^{2}} \delta^{(4)}(z-x) \delta^{(4)}(z-y), \\
\Gamma_{\rho(x) \sigma(y)}^{\sigma(z)}=\frac{1}{\rho(z)} \delta^{(4)}(z-x) \delta^{(4)}(z-y) .
\end{gathered}
$$

\section{a. Vilkovisky-DeWitt effective potential}

Let us first calculate the Vilkovisky-DeWitt effective action for this theory using (4.9). The covariant $2 \times 2$ inverse propagator is

$$
\nabla_{a} \nabla_{b} S=\left(\begin{array}{cc}
-\partial^{2}+\partial_{\mu} \sigma \partial^{\mu} \sigma-m^{2}-3 \lambda \rho^{2} & \rho \partial_{\mu} \sigma \partial^{\mu} \\
\rho \partial_{\mu} \sigma \partial^{\mu} & -\rho \partial_{\mu} \rho \partial^{\mu}-\rho^{2} \partial^{2}+\rho^{2} \partial_{\mu} \sigma \partial^{\mu} \sigma-m^{2} \rho^{2}-\lambda \rho^{4}
\end{array}\right) \delta\left(x_{I}-x_{J}\right),
$$

where $\phi^{a}=(\rho, \sigma)$. As before, we can use the $U(1)$ symmetry to set $\sigma=0$ without loss of generality. We will also consider a static configuration as before. Therefore, the one-loop VDW effective potential in the $\overline{\mathrm{MS}}$ scheme reads

$$
\begin{aligned}
V_{\mathrm{eff}}(\varphi) & \equiv-\frac{1}{V_{4}} \Gamma[\rho=\varphi, \sigma=0] \\
& =\frac{1}{2} m^{2} \varphi^{2}+\frac{\lambda}{4} \varphi^{4}-\ln \operatorname{det}\left[\partial^{2}+m^{2}+3 \lambda \varphi^{2}\right]-\ln \operatorname{det}\left[\partial^{2}+m^{2}+\lambda \varphi^{2}\right]-\ln \left(\varphi^{2}\right)+\ln \operatorname{det}\left[G_{a b}\right] \\
& =\frac{1}{2} m^{2} \varphi^{2}+\frac{1}{4} \lambda \varphi^{4}+\frac{1}{64 \pi^{2}}\left\{\left(m^{2}+3 \lambda \varphi^{2}\right)^{2}\left[\ln \left(\frac{m^{2}+3 \lambda \varphi^{2}}{\mu^{2}}\right)-\frac{3}{2}\right]+\left(m^{2}+\lambda \varphi^{2}\right)^{2}\left[\ln \left(\frac{m^{2}+\lambda \varphi^{2}}{\mu^{2}}\right)-\frac{3}{2}\right]\right\},
\end{aligned}
$$

where $V_{4}$ is the four-volume. Observe that the expression (A39) is identical to (A6). As expected, the VilkoviskyDeWitt effective action is independent of parametrization.

\section{b. Covariant Feynman rules and renormalization}

With the help of (5.19), we can calculate the covariant Feynman rules for this theory. We find them to be

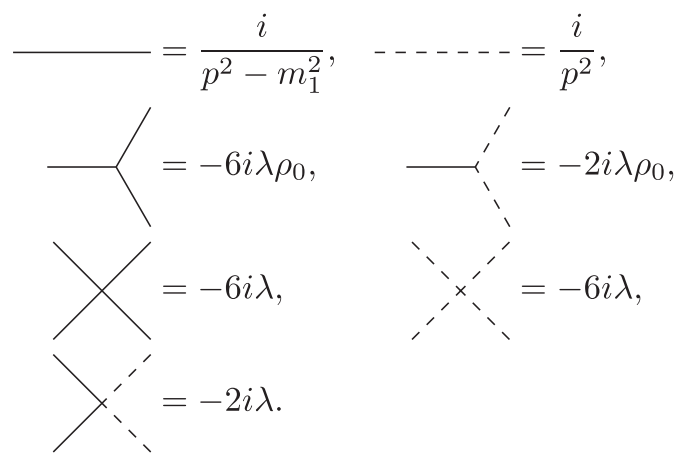

By construction, these Feynman rules are identical to (A7) and thus all RG calculation are identical both on and off shell.

\section{APPENDIX B: CURVED FIELD-SPACE EXAMPLE}

We wish to consider a simple toy model with genuine field-space curvature in order to study the effect this has on the quantum observables. Since it is impossible to have curvature in one dimension, we consider a theory with two fields $\rho$ and $\sigma$, and take $\sigma$ to be an angular variable with a shift symmetry. In order to avoid ghosts, the metric of the field space must be positive definite. Consequently, we take our field-space metric to be

$$
G_{A B}=\left(\begin{array}{cc}
1 & 0 \\
0 & \left(\rho / \rho_{0}\right)^{2 n}
\end{array}\right)
$$

The nonzero Christoffel symbols of this metric are easily calculated,

$$
\Gamma_{\sigma \sigma}^{\rho}=-n \frac{\rho^{2 n-1}}{\rho_{0}^{2 n}}, \quad \Gamma_{\rho \sigma}^{\sigma}=\frac{n}{\rho} .
$$

From these, we obtain the nonzero components of the fieldspace Riemann tensor,

$$
\begin{aligned}
& R_{\sigma \rho \sigma}^{\rho}=-\frac{n(n-1)}{\rho^{2}} \frac{\rho^{2 n}}{\rho_{0}^{2 n}}, \quad R_{\sigma \sigma \rho}^{\rho}=\frac{n(n-1)}{\rho^{2}} \frac{\rho^{2 n}}{\rho_{0}^{2 n}}, \\
& R_{\rho \rho \sigma}^{\sigma}=\frac{n(n-1)}{\rho^{2}}, \quad R_{\rho \sigma \rho}^{\sigma}=-\frac{n(n-1)}{\rho^{2}} .
\end{aligned}
$$

We see that provided $n \neq 0,1$, the Riemann tensor is nonzero and thus the field space is curved. Notice that $n=0$ and $n=1$ correspond to the two flat field-space examples we have looked at already in Appendix A 1 and A 2, respectively.

The simplest model with curvature is therefore the case $n=2$, which has the Lagrangian

$\mathcal{L}=\frac{1}{2} \partial_{\mu} \rho \partial^{\mu} \rho+\frac{1}{2}\left(\frac{\rho}{\rho_{0}}\right)^{4} \partial_{\mu} \sigma \partial^{\mu} \sigma-\frac{1}{2} m^{2} \rho^{2}-\frac{\lambda}{4} \rho^{4}$. 
We will consider the symmetry-broken vacuum,

$$
\langle\rho\rangle=\rho_{0} \equiv \sqrt{\frac{-m^{2}}{\lambda}}
$$

as we have done before.

\section{Standard approach}

We start by calculating the renormalization group flow in the standard way by looking at the standard Feynman rules (5.11). For the Lagrangian (B4), these are given by

$$
\begin{aligned}
& =\frac{i}{p^{2}-m_{1}^{2}}, \\
& =-6 i \lambda \rho_{0}, \\
& =-6 i \lambda,
\end{aligned}
$$$$
\ldots-\cdots=\frac{i}{p^{2}},
$$$$
\longrightarrow k_{2}^{k_{1}}=-4 i \frac{k_{1} \cdot k_{2}}{\rho_{0}} \text {, }
$$$$
k_{2} k_{1}=-12 i \frac{k_{3} \cdot k_{4}}{\rho_{0}^{2}} \text {, }
$$

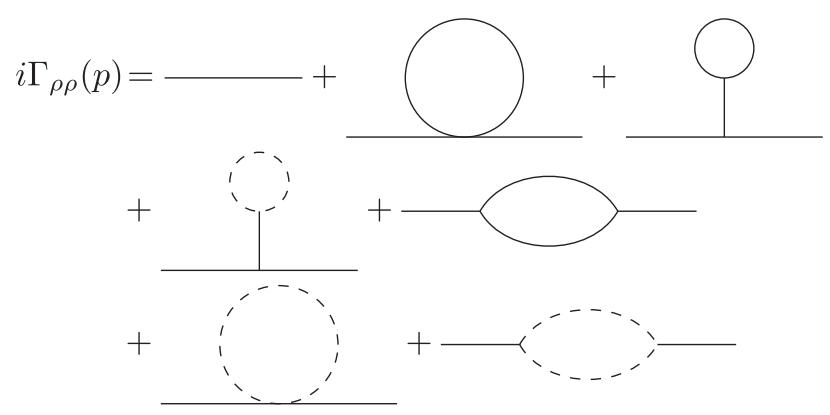

where $m_{1}^{2} \equiv m^{2}+3 \lambda \rho_{0}^{2}$ as before. Higher order interactions also exist; however, they will not be necessary for the oneloop calculations we will perform.

First, we will calculate the self-energy of the $\rho$ field. This is given schematically by

and calculated to be

$$
\begin{aligned}
i \Gamma_{\rho \rho}(p)= & i\left(p^{2}-m_{1}^{2}\right)+\frac{3 i \lambda}{(4 \pi)^{2}} A\left(m_{1}^{2}\right)-18 i \frac{\lambda^{2} \rho_{0}^{2}}{(4 \pi)^{2} m_{1}^{2}} A\left(m_{1}^{2}\right)+12 \frac{\lambda}{m_{1}^{2}} \int \frac{d^{4} k}{(2 \pi)^{4}}+18 i \frac{\lambda^{2} \rho_{0}^{2}}{(4 \pi)^{2}} B_{0}\left(p^{2}, m_{1}, m_{1}\right) \\
& -6 \frac{1}{\rho_{0}^{2}} \int \frac{d^{4} k}{(2 \pi)^{4}}+\frac{2 i p^{4}}{(4 \pi)^{2} \rho_{0}^{2}} B_{0}\left(p^{2}, 0,0\right) \\
= & i\left(p^{2}-m_{1}^{2}\right)+\frac{i \lambda m_{1}^{2}}{(4 \pi)^{2}}\left[\left(3+4 \frac{p^{4}}{m_{1}^{4}}\right) C_{\mathrm{UV}}+6 \ln \left(\frac{m_{1}^{2}}{\mu^{2}}\right)-4 \frac{p^{4}}{m_{1}^{4}} \ln \left(\frac{p^{2}}{\mu^{2}}\right)\right. \\
& \left.-9 \int_{0}^{1} d x \ln \left(\frac{m_{1}^{2}-x(1-x) p^{2}}{\mu^{2}}\right)-6+8 \frac{p^{4}}{m_{1}^{4}}\right] .
\end{aligned}
$$

As expected, the nonrenormalizability of the theory leads to a UV-divergent term proportional to $p^{4}$, which cannot be absorbed into a counterterm. In order to compare to the covariant approach, we calculate the on-shell self-energy as follows:

$\Gamma_{\rho \rho}\left(p^{2}=m_{1}^{2}\right)=\frac{\lambda m_{1}^{2}}{(4 \pi)^{2}}\left[-7 C_{\mathrm{UV}}-7 \ln \left(\frac{m_{1}^{2}}{\mu^{2}}\right)+20-3 \sqrt{3} \pi\right]$.

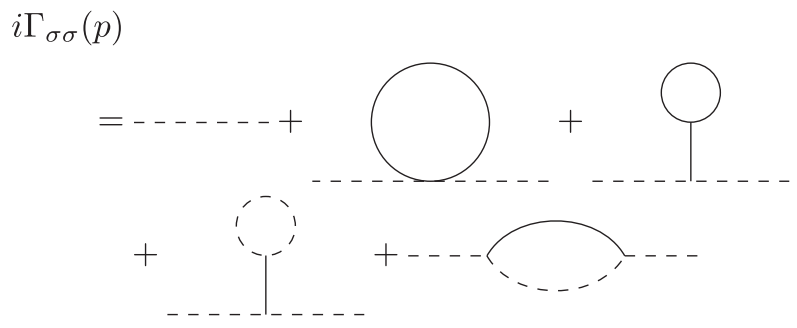

We now compare to the Goldstone self-energy, which is given schematically by

and calculated to be

$$
\begin{aligned}
i \Gamma_{\sigma \sigma}(p) & =i p^{2}+\frac{6 i p^{2}}{(4 \pi)^{2} \rho_{0}^{2}} A\left(m_{1}^{2}\right)-12 i \frac{p^{2} \lambda}{(4 \pi)^{2} m_{1}^{2}} A\left(m_{1}^{2}\right)-16 \frac{p^{2}}{\rho_{0}^{2} m_{1}^{2}} \int \frac{d^{4} k}{(2 \pi)^{4}}+4 i\left[\frac{3 p^{2}-m_{1}^{2}}{(4 \pi)^{2} \rho_{0}^{2}} A\left(m_{1}^{2}\right)+\frac{\left(p^{2}-m_{1}^{2}\right)^{2}}{(4 \pi)^{2} \rho_{0}^{2}} B_{0}\left(p^{4}, m_{1}, 0\right)\right] \\
& =i p^{2}+4 i \frac{3 p^{2}-m_{1}^{2}}{(4 \pi)^{2} \rho_{0}^{2}} m_{1}^{2}\left[C_{\mathrm{UV}}+1-\ln \left(\frac{m_{1}^{2}}{\mu^{2}}\right)\right]+4 i \frac{\left(p^{2}-m_{1}^{2}\right)^{2}}{(4 \pi)^{2} \rho_{0}^{2}}\left[C_{\mathrm{UV}}+1-\int_{0}^{1} d x \ln \left(\frac{x p^{2}-m_{1}^{2}}{\mu^{2}}\right)\right] .
\end{aligned}
$$


As before, due to the nonrenormalizability of the theory, this expression contains divergences that cannot be absorbed by a counterterm. However, on shell, we have $p^{2}=0$ and the expression reduces to

$$
\Gamma_{\sigma \sigma}\left(p^{2}=0\right)=0,
$$

implying that the Goldstone boson receives no correction to its mass as expected.

Finally, it is instructive to calculate the tree-level S-matrix element for $\rho \rho \rightarrow \sigma \sigma$. Taking into account the contributing diagrams, we find

$$
\begin{aligned}
& i \mathcal{M}(\rho \rho \rightarrow \sigma \sigma)= \\
& =-6 i \frac{s}{\rho_{0}^{2}}-6 i \frac{s m_{1}^{2}}{\rho_{0}^{2}\left(s-m_{1}^{2}\right)}-4 i \frac{\left(m_{1}^{2}-t\right)^{2}}{\rho_{0}^{2} t}-4 i \frac{\left(m_{1}^{2}-u\right)^{2}}{\rho_{0}^{2} u} \\
& =-\frac{2 i}{\rho_{0}^{2}}\left[3 \frac{s^{2}}{s-m_{1}^{2}}+2 \frac{\left(m_{1}^{2}-t\right)^{2}}{t}+2 \frac{\left(m_{1}^{2}-u\right)^{2}}{u}\right],
\end{aligned}
$$

where $s=\left(k_{1}+k_{2}\right)^{2}, t=\left(k_{1}-k_{3}\right)^{2}, u=\left(k_{1}-k_{4}\right)^{2}$ are the standard Mandelstam variables. Note that in the high energy limit, $\mathcal{M}(\rho \rho \rightarrow \sigma \sigma) \propto-2 s / \rho_{0}^{2}$.

\section{Covariant approach}

We now perform analogous calculations in the covariant formalism using (5.19). Here we show a limited set of the covariant Feynman rules for this theory,

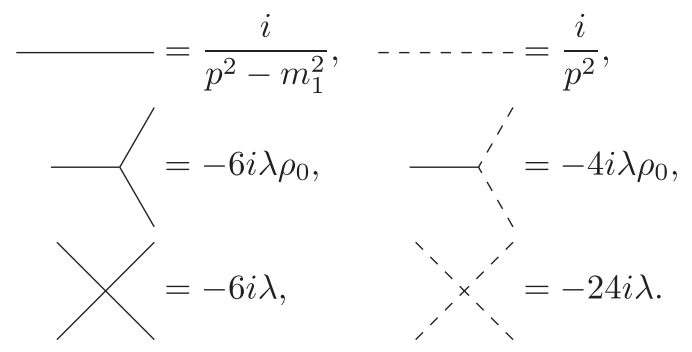

There are also an infinite set of higher order vertices, which we do not calculate since they do not affect the one-loop calculations we make in this section.

Finally, there is the $\rho \rho \sigma \sigma$ vertex. Due to the curvature of the field space, there is an ambiguity in the order in which the covariant derivatives are taken when calculating this vertex. In Sec. V, we argued that the correct approach was to symmetrize over all possible orderings. Nevertheless, we calculate each ordering explicitly. We have

$$
\begin{aligned}
& \sum_{k_{2}}^{k_{1}} \equiv \nabla_{\rho} \nabla_{\rho} \nabla_{\sigma} \nabla_{\sigma} S=-4 i \lambda-4 i \frac{k_{1} \cdot k_{2}}{\rho_{0}^{2}}, \\
& \rho \rho \sigma \sigma \\
& \sum_{\sigma \sigma \rho \rho}^{k_{1}} \equiv \nabla_{\sigma} \nabla_{\sigma} \nabla_{\rho} \nabla_{\rho} S=4 i \lambda-4 i \frac{k_{3} \cdot k_{4}}{\rho_{0}^{2}}, \\
& \sum_{\rho \sigma \rho \sigma, \rho \sigma \sigma \rho}^{k_{1}} \equiv \nabla_{\rho} \nabla_{\sigma} \nabla_{\rho} \nabla_{\sigma} S=2 i \frac{k_{1} \cdot\left(k_{3}+k_{4}-k_{2}\right)}{\rho_{0}^{2}}, \\
& \sum_{k_{2}}^{k_{1}} \equiv \nabla_{\sigma} \nabla_{\rho} \nabla_{\rho} \nabla_{\sigma} S=4 i \lambda+2 i \frac{k_{3} \cdot\left(k_{1}+k_{2}-k_{4}\right)}{\rho_{0}^{2}},
\end{aligned}
$$

where the ordering is denoted under the diagram. In the above, we did not display the other orderings of the two individual $\rho$ and $\sigma$ particles when calculating these rules, which may be obtained by exchanging $k_{1} \leftrightarrow k_{2}$ and/ or $k_{3} \leftrightarrow k_{4}$.

It is interesting to calculate the form of the vertices when taking all external particles to be on shell. We set

$$
k_{1}^{2}=k_{2}^{2}=m_{1}^{2}=2 \lambda \rho_{0}^{2}, \quad k_{3}^{2}=k_{4}^{2}=0 .
$$

Conservation of momentum then implies that

$$
\begin{aligned}
& 0=k_{1}+k_{2}+k_{3}+k_{4}, \quad k_{1} \cdot k_{2}=k_{3} \cdot k_{4}-m_{1}^{2}, \\
& k_{1} \cdot k_{3}=k_{2} \cdot k_{4}, \quad k_{1} \cdot k_{4}=k_{2} \cdot k_{3} .
\end{aligned}
$$

Employing these relations, we see that on shell, all six orderings are equal,

$$
\sum_{k_{2}}^{k_{1}}=k_{\sigma \rho \rho \rho}^{k_{4}}=k_{k_{3}}^{k_{4}}=4 i \lambda-4 i \frac{k_{3} \cdot k_{4}}{\rho_{0}^{2}} .
$$

Notice that the expression on (B18) is invariant under $k_{1} \leftrightarrow k_{2}$ and $k_{3} \leftrightarrow k_{4}$.

We have found that ordering does not matter when the particles are on shell. However, any quantum calculation will involve off-shell particles and for these, the ordering will make a difference. It is therefore important to use the fully symmetrized rule, 


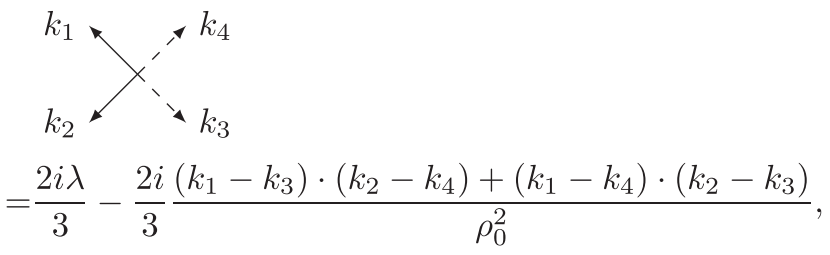

as discussed in Sec. V.

Let us now calculate the $\rho \rho$ and $\sigma \sigma$ self-energy as we did above. For the $\sigma \sigma$ self-energy, we obtain

$$
\begin{aligned}
i \Gamma_{\rho \rho}(p) & \\
= & i\left(p^{2}-m_{1}^{2}\right)+\frac{3 i \lambda}{(4 \pi)^{2}} A\left(m_{1}^{2}\right)-18 i \frac{\lambda^{2} \rho_{0}^{2}}{(4 \pi)^{2} m_{1}^{2}} A\left(m_{1}^{2}\right) \\
& +12 i \frac{\lambda^{2} \rho_{0}^{2}}{(4 \pi)^{2} m_{1}^{2}} A(0)+18 i \frac{\lambda^{2} \rho_{0}^{2}}{(4 \pi)^{2}} B_{0}\left(p^{2}, m_{1}, m_{1}\right) \\
& +\frac{i}{(4 \pi)^{2}} B_{0}\left(p^{2}, 0,0\right) \\
= & i\left(\frac{1}{(4 \pi)^{2}}\left(\frac{2}{3} \lambda+\frac{p^{2}}{\rho_{0}^{2}}\right) A(0)+\frac{1}{\rho_{0}^{2}} \int \frac{d^{4} k}{(2 \pi)^{4}}\right] \\
& -2+\frac{i \lambda m_{1}^{2}}{(4 \pi)^{2}}\left[7 C_{\mathrm{UV}}+6 \ln \left(\frac{m_{1}^{2}}{\mu^{2}}\right)+4 \ln \left(\frac{p^{2}}{\mu^{2}}\right)\right.
\end{aligned}
$$

Notice that in the covariant approach, there is no nonrenormalizable divergence. If we set the particle on- hell, we get

$\Gamma_{\rho \rho}\left(p^{2}=m_{1}^{2}\right)=\frac{\lambda m_{1}^{2}}{(4 \pi)^{2}}\left[7 C_{\mathrm{UV}}-7 \ln \left(\frac{m_{1}^{2}}{\mu^{2}}\right)+20-3 \sqrt{3} \pi\right]$.

This is in agreement with (B9).

In the previous calculation, only the final diagram of (B20) depends on the ordering of the Feynman rule and it vanishes regardless of the ordering. As such, the ordering was not really tested in this calculation. Let us instead calculate the self-energy of the $\sigma$ field, which will test the ordering. This is given by

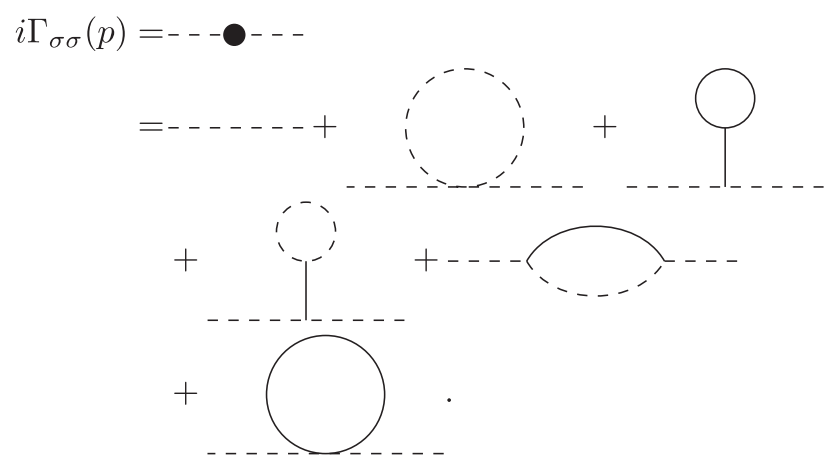

Before completing this calculation, let us focus on the last diagram, which is the only one in which the ordering makes a difference. Although we will eventually symmetrize over all possible orderings, let us first consider them all individually,

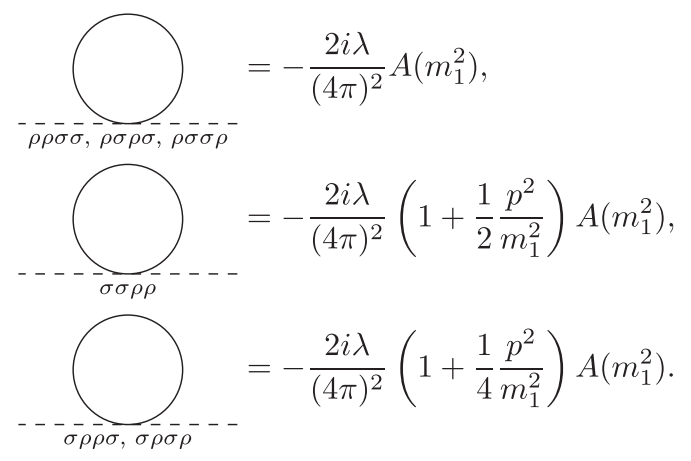

Note that the different orderings of the above diagrams lead to different results. However, these results converge to a single expression when the external particles are taken to be on shell, i.e $p^{2}=0$, despite the fact that the particle in the loop is off shell. For the diagram with off-shell external particles, we will use the symmetrized Feynman rule, which gives

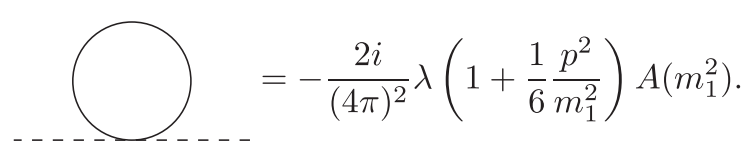

In this way, we find

$$
\begin{aligned}
\Gamma_{\sigma \sigma} & =p^{2}+\frac{12 \lambda}{(4 \pi)^{2}} A(0)-12 \frac{\lambda^{2} \rho_{0}^{2}}{(4 \pi)^{2} m_{1}^{2}} A\left(m_{1}^{2}\right)-8 \frac{\lambda^{2} \rho_{0}^{2}}{(4 \pi)^{2} m_{1}^{2}} A(0)+16 \frac{\lambda^{2} \rho_{0}^{2}}{(4 \pi)^{2}} B_{0}\left(p^{2}, m_{1}^{2}, 0\right)-\frac{2 \lambda}{(4 \pi)^{2}}\left(1+\frac{1}{6} \frac{p^{2}}{m_{1}^{2}}\right) A\left(m_{1}^{2}\right) \\
& =p^{2}\left(1-\frac{\lambda}{3} C_{\mathrm{UV}}\right)+\frac{8 \lambda m_{1}^{2}}{(4 \pi)^{2}}\left[\ln \left(\frac{m_{1}^{2}}{\mu^{2}}\right)-\int_{0}^{1} d x \ln \left(\frac{m_{1}^{2}-x p^{2}}{\mu^{2}}\right)+\frac{1}{24} \frac{p^{2}}{m_{1}^{2}}\left(\ln \left(\frac{m_{1}^{2}}{\mu^{2}}\right)-1\right)\right] .
\end{aligned}
$$


For on-shell Goldstone particles, we have

$$
\Gamma_{\sigma \sigma}\left(p^{2}=0\right)=0
$$

in agreement with (B12).

As in Appendix B 1, we calculate the tree-level S-matrix element for $\rho \rho \rightarrow \sigma \sigma$ in the covariant approach. The contributing diagrams are

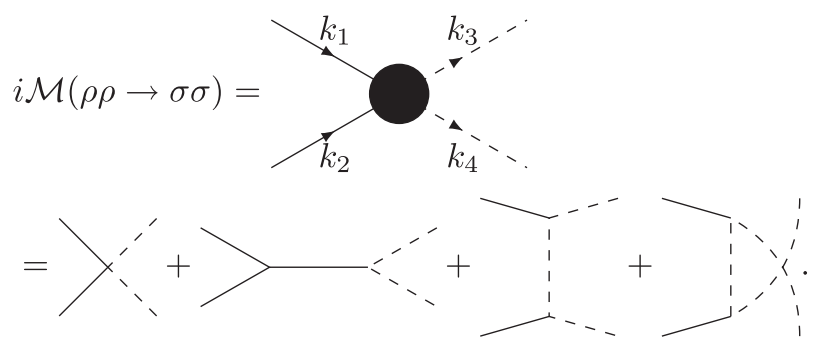

(B26)

As discussed earlier, the ordering in the first diagram does not matter when all particles are on shell. Therefore, we have

$$
\begin{gathered}
\mathcal{M}(\rho \rho \rightarrow \sigma \sigma) \\
=2\left(2 \lambda-\frac{s}{\rho_{0}^{2}}\right)-24 \lambda^{2} \rho_{0}^{2} \frac{1}{s-m_{1}^{2}}-16 \lambda^{2} \rho_{0}^{2} \frac{1}{t}-16 \lambda^{2} \rho_{0}^{2} \frac{1}{u} \\
=-\frac{2}{\rho_{0}^{2}}\left[3 \frac{s^{2}}{s-m_{1}^{2}}+2 \frac{\left(m_{1}^{2}-t\right)^{2}}{t}+2 \frac{\left(m_{1}^{2}-u\right)^{2}}{u}\right] \cdot \quad
\end{gathered}
$$

This result coincides with (B13).

\section{APPENDIX C: EXAMPLE WITH LINEAR POTENTIAL}

We now consider an example with Lagrangian given by

$\mathcal{L}=\frac{1}{2} \partial_{\mu} \rho \partial^{\mu} \rho+\frac{1}{2}\left(\frac{\rho}{\rho_{0}}\right)^{2} \partial_{\mu} \sigma \partial^{\mu} \sigma-t_{\rho} \rho-\frac{1}{2} m^{2} \rho^{2}$.

This has a flat field space - the kinetic part on its own is just a reparametrization of two canonical kinetic terms. Additionally, the potential has no interaction terms between the $\rho$ and $\sigma$ fields. However, as we shall see, the theory described by $(\mathrm{C} 1)$ is nonetheless interacting.

The theory has a symmetry-broken vacuum, which we parametrize as

$$
\langle\rho\rangle=\rho_{0} \equiv-t_{\rho} / m^{2}, \quad\langle\sigma\rangle=0 .
$$

We can then calculate the covariant Feynman rules for this theory using (5.19).

The propagators are

$$
\underline{\square}=\frac{i}{p^{2}-m^{2}}, \quad \ldots-\cdots=\frac{i}{p^{2}},
$$

where a solid line represents the Higgs mode $\rho$ and a dashed line represents the Goldstone mode $\sigma$.

The three- and four-point interactions are

$$
\begin{aligned}
& \square^{\prime}=\frac{i t_{\rho}}{\rho_{0}^{2}}, \\
& y^{\prime}{ }^{\prime}=-2 i \frac{t_{\rho}}{\rho_{0}^{3},},{ }^{\prime},{ }^{\prime}, \quad=3 i \frac{t_{\rho}}{\rho_{0}^{3}},
\end{aligned}
$$

while the five- and six-point interactions are

$$
\sum_{\vdots}^{i}=6 i \frac{t_{\rho}}{\rho_{0}^{4}}, \quad \begin{gathered}
i \\
\vdots
\end{gathered}=-24 i \frac{t_{\rho}}{\rho_{0}^{5}}, \quad \vdots
$$

Notice that there is an infinite series of higher-point vertices, which are proportional to $t_{\rho}$. Moreover, these infinite series include interactions that are absent in the standard approach.

To better understand why this theory has an infinite tower of interactions, we switch to a canonical parametrization and define

$$
\phi_{1}=\rho \cos \left(\frac{\sigma}{\rho_{0}}\right), \quad \phi_{2}=\rho \sin \left(\frac{\sigma}{\rho_{0}}\right) .
$$

Then, $(\mathrm{C} 1)$ takes the form

$$
\begin{aligned}
\mathcal{L}= & \frac{1}{2} \partial_{\mu} \phi_{1} \partial^{\mu} \phi_{1}+\frac{1}{2} \partial_{\mu} \phi_{2} \partial^{\mu} \phi_{2}-\frac{1}{2} m^{2}\left(\phi_{1}^{2}+\phi_{2}^{2}\right) \\
& -t_{\rho} \sqrt{\phi_{1}^{2}+\phi_{2}^{2}} .
\end{aligned}
$$

With this parametrization, the field-space metric becomes manifestly Euclidean and thus ordinary and covariant Feynman rules will be identical. The final term in (C7) is nonpolynomial and thus has an infinite Taylor series expansion. This term therefore leads to an infinite tower of Feynman rules as presented. 


\section{APPENDIX D: FIELD-SPACE RIEMANN TENSOR FOR GENERAL RELATIVITY}

In Sec. VI, we have only presented the field-space Ricci tensor and Ricci scalar, but not the full expression for the Riemann tensor $\mathfrak{R}^{(\mu \nu)}{ }_{(\alpha \beta)(\rho \sigma)(\gamma \delta)}$ due to its length. In this Appendix, we explicitly display $\mathfrak{R}^{(\mu \nu)}{ }_{(\alpha \beta)(\rho \sigma)(\gamma \delta)}$. With the aid of the symbolic computer algebra system CADABRA2 [70,71], we find that the field-space Riemann tensor for general relativity reads

$$
\begin{aligned}
\mathfrak{R}_{(\alpha \nu)(\rho \sigma)(\gamma \delta)=}^{(\mu \nu)} & -\frac{1}{32} \delta_{\rho}^{\mu} \delta_{\beta}^{\nu} g_{\sigma \gamma} g_{\alpha \delta}-\frac{1}{32} \delta_{\sigma}^{\mu} \delta_{\beta}^{\nu} g_{\rho \gamma} g_{\alpha \delta}-\frac{1}{32} \delta_{\beta}^{\mu} \delta_{\sigma}^{\nu} g_{\rho \gamma} g_{\alpha \delta}-\frac{1}{32} \delta_{\beta}^{\mu} \delta_{\rho}^{\nu} g_{\sigma \gamma} g_{\alpha \delta} \\
& -\frac{1}{32} \delta_{\rho}^{\mu} \delta_{\beta}^{\nu} g_{\sigma \delta} g_{\alpha \gamma}-\frac{1}{32} \delta_{\sigma}^{\mu} \delta_{\beta}^{\nu} g_{\rho \delta} g_{\alpha \gamma}-\frac{1}{32} \delta_{\beta}^{\mu} \delta_{\sigma}^{\nu} g_{\rho \delta} g_{\alpha \gamma}-\frac{1}{32} \delta_{\beta}^{\mu} \delta_{\rho}^{\nu} g_{\sigma \delta} g_{\alpha \gamma} \\
& -\frac{1}{32} \delta_{\alpha}^{\mu} \delta_{\rho}^{\nu} g_{\sigma \gamma} g_{\beta \delta}-\frac{1}{32} \delta_{\alpha}^{\mu} \delta_{\sigma}^{\nu} g_{\rho \gamma} g_{\beta \delta}-\frac{1}{32} \delta_{\rho}^{\mu} \delta_{\alpha}^{\nu} g_{\sigma \gamma} g_{\beta \delta}-\frac{1}{32} \delta_{\sigma}^{\mu} \delta_{\alpha}^{\nu} g_{\rho \gamma} g_{\beta \delta} \\
& -\frac{1}{32} \delta_{\alpha}^{\mu} \delta_{\rho}^{\nu} g_{\sigma \delta} g_{\beta \gamma}-\frac{1}{32} \delta_{\alpha}^{\mu} \delta_{\sigma}^{\nu} g_{\rho \delta} g_{\beta \gamma}-\frac{1}{32} \delta_{\rho}^{\mu} \delta_{\alpha}^{\nu} g_{\sigma \delta} g_{\beta \gamma}-\frac{1}{32} \delta_{\sigma}^{\mu} \delta_{\alpha}^{\nu} g_{\rho \delta} g_{\beta \gamma} \\
& +\frac{1}{32} \delta_{\gamma}^{\mu} \delta_{\beta}^{\nu} g_{\rho \delta} g_{\sigma \alpha}+\frac{1}{32} \delta_{\delta}^{\mu} \delta_{\beta}^{\nu} g_{\rho \gamma} g_{\sigma \alpha}+\frac{1}{32} \delta_{\beta}^{\mu} \delta_{\delta}^{\nu} g_{\rho \gamma} g_{\sigma \alpha}+\frac{1}{32} \delta_{\beta}^{\mu} \delta_{\gamma}^{\nu} g_{\rho \delta} g_{\sigma \alpha} \\
& +\frac{1}{32} \delta_{\gamma}^{\mu} \delta_{\beta}^{\nu} g_{\rho \alpha} g_{\sigma \delta}+\frac{1}{32} \delta_{\delta}^{\mu} \delta_{\beta}^{\nu} g_{\rho \alpha} g_{\sigma \gamma}+\frac{1}{32} \delta_{\beta}^{\mu} \delta_{\delta}^{\nu} g_{\rho \alpha} g_{\sigma \gamma}+\frac{1}{32} \delta_{\beta}^{\mu} \delta_{\gamma}^{\nu} g_{\rho \alpha} g_{\sigma \delta} \\
& +\frac{1}{32} \delta_{\alpha}^{\mu} \delta_{\gamma}^{\nu} g_{\rho \delta} g_{\sigma \beta}+\frac{1}{32} \delta_{\alpha}^{\mu} \delta_{\delta}^{\nu} g_{\rho \gamma} g_{\sigma \beta}+\frac{1}{32} \delta_{\gamma}^{\mu} \delta_{\alpha}^{\nu} g_{\rho \delta} g_{\sigma \beta}+\frac{1}{32} \delta_{\delta}^{\mu} \delta_{\alpha}^{\nu} g_{\rho \gamma} g_{\sigma \beta} \\
& +\frac{1}{32} \delta_{\alpha}^{\mu} \delta_{\gamma}^{\nu} g_{\rho \beta} g_{\sigma \delta}+\frac{1}{32} \delta_{\alpha}^{\mu} \delta_{\delta}^{\nu} g_{\rho \beta} g_{\sigma \gamma}+\frac{1}{32} \delta_{\gamma}^{\mu} \delta_{\alpha}^{\nu} g_{\rho \beta} g_{\sigma \delta}+\frac{1}{32} \delta_{\delta}^{\mu} \delta_{\alpha}^{\nu} g_{\rho \beta} g_{\sigma \gamma} \\
& +\frac{1}{4 D} g_{\rho \gamma} g^{\mu \nu} g_{\sigma \beta} g_{\alpha \delta}+\frac{1}{4 D} g_{\rho \delta} g^{\mu \nu} g_{\sigma \beta} g_{\alpha \gamma}+\frac{1}{4 D} g_{\rho \alpha} g^{\mu \nu} g_{\sigma \gamma} g_{\beta \delta}+\frac{1}{4 D} g_{\rho \alpha} g^{\mu \nu} g_{\sigma \delta} g_{\beta \gamma} \\
& +\frac{1}{4 D} g_{\rho \gamma} g^{\mu \nu} g_{\sigma \alpha} g_{\beta \delta}+\frac{1}{4 D} g_{\rho \delta} g^{\mu \nu} g_{\sigma \alpha} g_{\beta \gamma}+\frac{1}{4 D} g_{\rho \beta} g^{\mu \nu} g_{\sigma \delta} g_{\alpha \gamma}+\frac{1}{4 D} g_{\rho \beta} g^{\mu \nu} g_{\sigma \gamma} g_{\alpha \delta} \\
& -\frac{1}{4 D} g^{\mu \nu} g_{\rho \beta} g_{\sigma \gamma} g_{\alpha \delta}-\frac{1}{4 D} g^{\mu \nu} g_{\rho \beta} g_{\sigma \delta} g_{\alpha \gamma}-\frac{1}{4 D} g^{\mu \nu} g_{\rho \gamma} g_{\sigma \alpha} g_{\beta \delta}-\frac{1}{4 D} g^{\mu \nu} g_{\rho \delta} g_{\sigma \alpha} g_{\beta \gamma} \\
& -\frac{1}{4 D} g^{\mu \nu} g_{\rho \alpha} g_{\sigma \gamma} g_{\beta \delta}-\frac{1}{4 D} g^{\mu \nu} g_{\rho \alpha} g_{\sigma \delta} g_{\beta \gamma}-\frac{1}{4 D} g^{\mu \nu} g_{\rho \delta} g_{\sigma \beta} g_{\alpha \gamma}-\frac{1}{4 D} g^{\mu \nu} g_{\rho \gamma} g_{\sigma \beta} g_{\alpha \delta} .
\end{aligned}
$$

We note that this tensor vanishes for $D=1$. This is to be expected since the field space of gravity in one dimension cannot be anything other than trivial.

Note that these results differ from those reported in [81], where the DeWitt metric was used instead.

[1] A. Einstein, The field equations of gravitation, Sitzungsber. Preuss. Akad. Wiss. Berlin (Math. Phys.) 1915, 844 (1915).

[2] M. Fierz, On the physical interpretation of P. Jordan's extended theory of gravitation, Helv. Phys. Acta 29, 128 (1956).

[3] A. O. Barvinsky and G. A. Vilkovisky, The generalized Schwinger-Dewitt technique in gauge theories and quantum gravity, Phys. Rep. 119, 1 (1985).

[4] S. Capozziello, R. de Ritis, and A. A. Marino, Some aspects of the cosmological conformal equivalence between 'Jordan frame' and 'Einstein frame', Classical Quantum Gravity 14, 3243 (1997).
[5] V. Faraoni, E. Gunzig, and P. Nardone, Conformal transformations in classical gravitational theories and in cosmology, Fundam. Cosm. Phys. 20, 121 (1999).

[6] E. Alvarez and J. Conde, Are the string and Einstein frames equivalent, Mod. Phys. Lett. A 17, 413 (2002).

[7] S. Capozziello, P. Martin-Moruno, and C. Rubano, Physical non-equivalence of the Jordan and Einstein frames, Phys. Lett. B 689, 117 (2010).

[8] C. F. Steinwachs and A. Y. Kamenshchik, Non-minimal Higgs inflation and frame dependence in cosmology, AIP Conf. Proc. 1514, 161 (2013). 
[9] L. Järv, P. Kuusk, M. Saal, and O. Vilson, Invariant quantities in the scalar-tensor theories of gravitation, Phys. Rev. D 91, 024041 (2015).

[10] A. Y. Kamenshchik and C. F. Steinwachs, Question of quantum equivalence between Jordan frame and Einstein frame, Phys. Rev. D 91, 084033 (2015).

[11] M. Postma and M. Volponi, Equivalence of the Einstein and Jordan frames, Phys. Rev. D 90, 103516 (2014).

[12] G. Doménech and M. Sasaki, Conformal frame dependence of inflation, J. Cosmol. Astropart. Phys. 04 (2015) 022.

[13] L. Järv, K. Kannike, L. Marzola, A. Racioppi, M. Raidal, M. Rünkla, M. Saal, and H. Veermäe, Frame-Independent Classification of Single-Field Inflationary Models, Phys. Rev. Lett. 118, 151302 (2017).

[14] M. Herrero-Valea, Anomalies, equivalence and renormalization of cosmological frames, Phys. Rev. D 93, 105038 (2016).

[15] S. Pandey and N. Banerjee, Equivalence of Jordan and Einstein frames at the quantum level, Eur. Phys. J. Plus 132, 107 (2017).

[16] A. Karam, A. Lykkas, and K. Tamvakis, Frame-invariant approach to higher-dimensional scalar-tensor gravity, Phys. Rev. D 97, 124036 (2018).

[17] K. Falls and M. Herrero-Valea, Frame (In)equivalence in quantum field theory and cosmology, Eur. Phys. J. C 79, 595 (2019).

[18] D. Nandi and P. Saha, Einstein or Jordan: Seeking answers from the reheating constraints, arXiv:1907.10295.

[19] J. Francfort, B. Ghosh, and R. Durrer, Cosmological number counts in Einstein and Jordan frames, J. Cosmol. Astropart. Phys. 09 (2019) 071.

[20] S. Karamitsos and A. Pilaftsis, On the cosmological frame problem, Proc. Sci., CORFU2017 (2018) 036.

[21] P. Jordan, Zur empirischen Kosmologie, Naturwissenschaften 26, 417 (1938).

[22] P. Jordan, Schwerkraft und Weltall (Die Wissenschaft, Bd. 107, Braunschweig, 1952).

[23] C. Brans and R. H. Dicke, Mach's principle and a relativistic theory of gravitation, Phys. Rev. 124, 925 (1961).

[24] P. G. Bergmann, Comments on the scalar tensor theory, Int. J. Theor. Phys. 1, 25 (1968).

[25] R. V. Wagoner, Scalar tensor theory and gravitational waves, Phys. Rev. D 1, 3209 (1970).

[26] Y. Fujii and K. Maeda, The Scalar-Tensor Theory of Gravitation, Cambridge Monographs on Mathematical Physics (Cambridge University Press, Cambridge, United Kingdom, 2007).

[27] V. Faraoni, Cosmology in Scalar Tensor Gravity, Fundamental Theories of Physics Vol. 139 (Springer, Dordrecht, 2004).

[28] S. Karamitsos and A. Pilaftsis, Frame covariant nonminimal multifield inflation, Nucl. Phys. B927, 219 (2018).

[29] G. A. Vilkovisky, The unique effective action in quantum field theory, Nucl. Phys. B234, 125 (1984).

[30] S. Groot Nibbelink and B. J. W. van Tent, Density perturbations arising from multiple field slow roll inflation, arXiv: hep-ph/0011325.

[31] S. Groot Nibbelink and B. J. W. van Tent, Scalar perturbations during multiple field slow-roll inflation, Classical Quantum Gravity 19, 613 (2002).
[32] B. van Tent, Multiple-field inflation and the CMB, Classical Quantum Gravity 21, 349 (2004).

[33] D. Burns, S. Karamitsos, and A. Pilaftsis, Frame-covariant formulation of inflation in scalar-curvature theories, Nucl. Phys. B907, 785 (2016).

[34] C. F. Steinwachs, Non-Minimal Higgs Inflation and Frame Dependence in Cosmology (Springer, Cham, 2014).

[35] F. Sauter, Uber das Verhalten eines Elektrons im homogenen elektrischen Feld nach der relativistischen Theorie Diracs, Z. Phys. 69, 742 (1931).

[36] W. Heisenberg and H. Euler, Consequences of Dirac's theory of positrons, Z. Phys. 98, 714 (1936).

[37] V. Weisskopf, The electrodynamics of the vacuum based on the quantum theory of the electron, Kong. Dan. Vid. Sel. Mat. Fys. Med. 14, 1 (1936).

[38] J. S. Schwinger, On gauge invariance and vacuum polarization, Phys. Rev. 82, 664 (1951).

[39] G. A. Vilkovisky, The Gospel According to Dewitt, edited by S. M. Christensen, Quantum Theory of Gravity (Adam Hilger Limited, Bristol, 1984), pp. 169-209.

[40] The effective action, Architecture of Fundamental Interactions at Short Distances: Proceedings, Les Houches 44th Summer School of Theoretical Physics: Les Houches, France, 1985, pt2 (Elsevier Science Pub. Co., New York, 1987), pp. 1023-1058.

[41] B. S. DeWitt, Quantum theory of gravity. 2. The manifestly covariant theory, Phys. Rev. 162, 1195 (1967).

[42] P. Finsler, Über Kurven und Flächen in allgemeinen Räumen (Göttingen, Zürich: O. Füssli, 120 S. $8^{\circ}$ (1918).

[43] L. F. Abbott, Introduction to the background field method, Acta Phys. Pol. B 13, 33 (1982).

[44] A. Rebhan, The Vilkovisky-de Witt effective action and its application to Yang-Mills theories, Nucl. Phys. B288, 832 (1987).

[45] S. R. Huggins, G. Kunstatter, H. P. Leivo, and D. J. Toms, On the Unique Effective Action in Five-dimensional Kaluza-Klein Theory, Phys. Rev. Lett. 58, 296 (1987).

[46] G. Kunstatter, Vilkovisky's Unique effective action: An introduction and explicit calculation, in Vancouver 1986, Proceedings, Super Field Theories, 503-517 and preprint-G. Kunstatter (Plenum, New York, 1986), p. 28.

[47] G. Kunstatter, Geometrical approach to the effective action, Report No. LPTHE-ORSAY-90-43.

[48] P. Ellicott and D. J. Toms, On the new effective action in quantum field theory, Nucl. Phys. B312, 700 (1989).

[49] C. P. Burgess and G. Kunstatter, On the physical interpretation of the Vilkovisky-de Witt effective action, Mod. Phys. Lett. A 02, 875 (1987); Erratum, Mod. Phys. Lett. A 02, 1003 (1987).

[50] M. F. Sohnius, Introducing supersymmetry, Phys. Rep. 128, 39 (1985).

[51] D. R. Grigore, Off-shell fields and quantum anomalies, Ann. U. Craiova Phys. 21, S117 (2010).

[52] A. R. Liddle, P. Parsons, and J. D. Barrow, Formalizing the slow roll approximation in inflation, Phys. Rev. D 50, 7222 (1994).

[53] J. Honerkamp, Chiral multiloops, Nucl. Phys. B36, 130 (1972). 
[54] G. Ecker and J. Honerkamp, Covariant perturbation theory and chiral superpropagators, Phys. Lett. B 42, 253 (1972).

[55] J. Honerkamp, F. Krause, and M. Scheunert, On the equivalence of standard and covariant perturbation series in non-polynomial pion lagrangian field theory, Nucl. Phys. B69, 618 (1974).

[56] H. Lehmann, K. Symanzik, and W. Zimmermann, On the formulation of quantized field theories, Nuovo Cimento 1, 205 (1955).

[57] S. Pokorski, Gauge Field Theories, 2nd ed. (Cambridge University Press, Cambridge, Englnad, 2000).

[58] M. Srednicki, Quantum Field Theory (Cambridge University Press, Cambridge, United Kingdom, 2007).

[59] L. H. Ryder, Quantum Field Theory (Cambridge University Press, Cambridge, United Kingdom, 1996).

[60] R. P. Feynman, The theory of positrons, Phys. Rev. 76, 749 (1949).

[61] B. W. Lee, Gauge theories, Conf. Proc. C 7507281, 79 (1975).

[62] M. Bounakis and I. G. Moss, Gravitational corrections to Higgs potentials, J. High Energy Phys. 04 (2018) 071.

[63] G. 't Hooft and M. J. G. Veltman, One loop divergencies in the theory of gravitation, Ann. Inst. Henri Poincare Phys. Theor. A 20, 69 (1974).

[64] M. H. Goroff and A. Sagnotti, Quantum gravity at two loops, Phys. Lett. 160B, 81 (1985).

[65] S. D. Odintsov, Does the Vilkovisky-De Witt effective action in quantum gravity depend on the configuration space metric?, Phys. Lett. B 262, 394 (1991).

[66] D. Giulini and C. Kiefer, Wheeler-DeWitt metric and the attractivity of gravity, Phys. Lett. A 193, 21 (1994).

[67] B. S. DeWitt, Quantum theory of gravity. 1. The canonical theory, Phys. Rev. 160, 1113 (1967).
[68] B. S. DeWitt, A gauge invariant effective action, CERN Report No. NSF-ITP-80-31, 1980.

[69] E. Fradkin and A. A. Tseytlin, On the new definition of offshell effective action, Nucl. Phys. B234, 509 (1984).

[70] K. Peeters, Introducing cadabra: A symbolic computer algebra system for field theory problems, arXiv:hep-th/ 0701238.

[71] K. Peeters, Cadabra2: Computer algebra for field theory revisited, J. Open Source Software 3, 1118 (2018).

[72] N. Ohta, Quantum equivalence of $f(R)$ gravity and scalartensor theories in the Jordan and Einstein frames, Prog. Theor. Exp. Phys. 2018, 033B02 (2018).

[73] E. Buckingham, On physically similar systems; illustrations of the use of dimensional equations, Phys. Rev. 4, 345 (1914).

[74] P. W. Higgs, Quadratic lagrangians and general relativity, Nuovo Cimento 11, 816 (1959).

[75] E. E. Flanagan, The conformal frame freedom in theories of gravitation, Classical Quantum Gravity 21, 3817 (2004).

[76] R. Catena, M. Pietroni, and L. Scarabello, Einstein and Jordan reconciled: A frame-invariant approach to scalartensor cosmology, Phys. Rev. D 76, 084039 (2007).

[77] K. Fujikawa, Path integral measure for gravitational interactions, Nucl. Phys. B226, 437 (1983).

[78] L. Faddeev and V. Popov, Feynman diagrams for the YangMills field, Phys. Lett. 25B, 29 (1967).

[79] C. G. Callan, Jr., Broken scale invariance in scalar field theory, Phys. Rev. D 2, 1541 (1970).

[80] K. Symanzik, Small distance behavior in field theory and power counting, Commun. Math. Phys. 18, 227 (1970).

[81] C. F. Steinwachs and M. L. van der Wild, Quantum gravitational corrections from the Wheeler-DeWitt equation for scalartensor theories, Classical Quantum Gravity 35, 135010 (2018). 